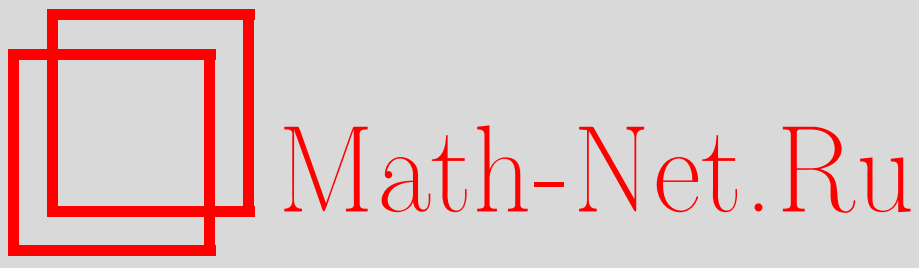

А. Б. Шишкин, Спектральный синтез для систем дифференциальных операторов с постоянными коэффициентами, Матем. сб., 2003, том 194, номер 12, 123-156

DOI: https://doi.org/10.4213/sm789

Использование Общероссийского математического портала Math-Net.Ru подразумевает, что вы прочитали и согласны с пользовательским соглашением http://www . mathnet.ru/rus/agreement

Параметры загрузки:

IP: 54.162 .127 .20

26 апреля 2023 г., 13:32:20 
УДК 517.5

А.Б. Шишкин

\title{
Спектральный синтез для систем дифференциальных операторов с постоянными коэффициентами
}

\begin{abstract}
Рассмотрены подпространства аналитических функций одной комплексной переменной, инвариантные относительно систем дифференциальных операторов с постоянньми коэффициентами. Описьваются ситуации, когда такие инвариантные подпространства допускают спектральньй синтез.

Библиография: 25 названий.
\end{abstract}

Настоящая статья использует терминологию и обозначения статей [1]-[3].

\section{§1. Введение}

1.1. Спектральный синтез. Выделим в кольце $\mathbb{C}[z]$ многочленов от одной переменной совокупность из $q$ элементов $\pi_{1}, \ldots, \pi_{q}$. Условимся отождествлять в обозначениях многочлены и осушествляемые ими отображения. Следуя этому соглашению, символом $\pi$ будем обозначать $q$-многочлен $\left(\pi_{1}, \ldots, \pi_{q}\right)$ и отображение из $\mathbb{C}$ в $\mathbb{C}^{q}$, осуществляемое этим $q$-многочленом. Обозначим через $\Lambda$ образ $\mathbb{C}$ при отображении $\pi$. Считаем, что среди многочленов $\pi_{1}, \ldots, \pi_{q}$ есть хотя бы один, отличньй от константы. При этом условии отображение $\pi$ имеет конечные слои

$$
\widetilde{\lambda}:=\pi^{-1}(\lambda)=\bigcap_{p=1}^{q} \pi_{p}^{-1}\left(\lambda_{p}\right), \quad \lambda=\left(\lambda_{1}, \ldots, \lambda_{q}\right) \in \Lambda .
$$

Если $q>1$, то $\Lambda$ - собственное подмножество $\mathbb{C}^{q}$.

Пусть $\Omega=\left\{\Omega_{1}, \ldots, \Omega_{\nu}\right\}$ - некоторая система односвязных областей в $\mathbb{C} ; H_{j}=$ $H\left(\Omega_{j}\right)$ - пространство функций, голоморфных в $\Omega_{j}$, с топологией компактной сходимости; $\mathbf{H}$ - топологическое произведение $H_{1}, \ldots, H_{\nu} ; D$ - оператор дифференцирования, действуюший в Н покомпонентно: $D(f)=\left(f_{1}^{\prime}, \ldots, f_{\nu}^{\prime}\right), f=\left(f_{1}, \ldots, f_{\nu}\right) \in$ $\mathbf{H}$;

$$
\pi_{1}(D), \ldots, \pi_{q}(D)
$$

- система линейных дифференциальных операторов с постоянньми коэффициентами.

Отличный от нуля элемент $f \in \mathbf{H}$ называется корневым әлементом системы операторов (1), отвечающим вектору собственных значений $\lambda=\left(\lambda_{1}, \ldots, \lambda_{q}\right) \in$ $\mathbb{C}^{q}$, если он при некотором натуральном $k$ удовлетворяет системе уравнений

$$
\left(\pi_{1}(D)-\lambda_{1}\right)^{k} f=0, \quad \ldots, \quad\left(\pi_{q}(D)-\lambda_{q}\right)^{k} f=0 .
$$

Легко показать, что запас всех векторов собственных значений системы операторов (1) совпадает с $\Lambda$. При этом всякий корневой элемент системы операторов (1), 
отвечающий вектору собственных значений $\lambda \in \Lambda$, представляется в виде конечной линейной комбинации

$$
\sum a_{n, \omega, j} z^{n} \exp _{j} \omega z, \quad n \in \mathbb{Z}_{+}, \quad \omega \in \tilde{\lambda}, \quad j=1, \ldots, \nu,
$$

с коэффициентами $a_{n, \omega, j}$ из $\mathbb{C}$, где $\exp _{j} \omega z:=e_{j} \exp \omega z, e_{1}, \ldots, e_{\nu}-$ система единичных векторов.

Подпространство $W \subseteq \mathbf{H}$ называем инвариантным, если оно инвариантно относительно каждого из операторов системы (1). Говорят, что замкнутое инвариантное подпространство $W \subseteq \mathbf{H}$ допускает спектральный синтез, если оно совпадает с замыканием в Н линейной оболочки корневых элементов системы операторов (1), содержащихся в $W$. Задача спектрального синтеза для системы операторов (1) состоит в нахождении условий, при которых замкнутое инвариантное подпространство $W \subseteq \mathbf{H}$ допускает спектральньй синтез.

1.2. Локальное описание. Пусть $H_{j}^{*}$ - сильное сопряженное к пространству $H_{j} ; T_{j}$ - преобразование Лапласа, которое каждому функционалу $S \in H_{j}^{*}$ ставит в соответствие целую функцию экспоненциального типа $\varphi(\zeta)=\left\langle S, e^{\zeta z}\right\rangle$; $P_{j}=T_{j}\left(H_{j}^{*}\right)$ - полньй образ отображения $T_{j}$. Отображение $T_{j}: H_{j}^{*} \rightarrow P_{j}$ является взаимно однозначньп; оно индуцирует в $P_{j}$ отделимую локально выпуклую топологию. Известно, что $P_{j}$ - топологический модуль над кольцом многочленов $\mathbb{C}[\zeta]$.

Сильное сопряженное $\mathbf{H}^{*}$ к пространству $\mathbf{H}$ совпадает с топологическим произведением $H_{1}^{*} \times \cdots \times H_{\nu}^{*}$. Пусть $\mathbf{P}$ - топологическое произведение $P_{1} \times \cdots \times P_{\nu}$, $\mathbf{T}$ - отображение $\mathbf{H}^{*} \rightarrow \mathbf{P},\left(S_{1}, \ldots, S_{\nu}\right) \rightarrow\left(T_{1}\left(S_{1}\right), \ldots, T_{\nu}\left(S_{\nu}\right)\right)$. Пространство $\mathbf{P}$ наследует основные свойства пространств $P_{1}, \ldots, P_{\nu}$. В частности, отображение $\mathbf{H}^{*} \rightarrow \mathbf{P}$ является линейным топологическим изоморфизмом, $\mathbf{P}$ выдерживает покомпонентное умножение на многочлены и это умножение непрерывно в топологии Р. Кроме того, в пространстве $\mathbf{P}$ выполняется импликация устойчивости:

$$
\varphi \in \mathbf{P}, \quad \zeta-a \mid \varphi \quad \Rightarrow \quad \frac{\varphi}{\zeta-a} \in \mathbf{P}
$$

Здесь $\zeta-a \mid \varphi$ означает, что двучлен $\zeta-a$ делит каждую компоненту элемента $\varphi$.

Кольцо $\mathbb{C}[\pi]:=\mathbb{C}\left[\pi_{1}, \ldots, \pi_{q}\right]$ многочленов от $\pi_{1}, \ldots, \pi_{q}$ является подкольцом кольца $\mathbb{C}[\zeta]$. Это позволяет рассматривать $\mathbf{P}$ как модуль над кольцом $\mathbb{C}[\pi]$. Известно, что этот модуль является топологическим [4; $\S 2]$. Следовательно, замькание $\mathbb{C}[\pi]$-подмодуля в $\mathbf{P}$ есть снова $\mathbb{C}[\pi]$-подмодуль в $\mathbf{P}$.

Множество $U \subseteq \mathbb{C}$ называется $\pi$-симметричным, если найдется множество $V \subseteq \mathbb{C}^{q}$ такое, что $U=\pi^{-1}(V)$. Функция $\varphi$, голоморфная в открытом $\pi$-симметричном множестве, называется $\pi$-симметричной, если для каждого $\pi$-слоя $\tilde{\lambda}$ из области определения функции $\varphi$ найдутся открытая $\pi$-симметричная окрестность $U$ этого слоя и открытая окрестность $V \subseteq \mathbb{C}^{q}$ точки $\lambda=\pi(\widetilde{\lambda})$, содержащая $\pi(U)$, такие, что сужение $\varphi$ на $U$ представляется в виде $\Phi$ о $\pi$, где $\Phi$ - некоторая функция, голоморфная в $V$. Простейшие $\pi$-симметричные функции - это отображения, осуществляемые элементами кольца $\mathbb{C}[\pi]$.

Топологию, которую образуют в $\mathbb{C}$ открытые $\pi$-симметричные множества, обозначим через $\tau_{\pi}$. Пусть $U \in \tau_{\pi} ; O(U)$ - кольцо голоморфных на $U$ функций; $\mathbf{O}(U)-$ декартово произведение $\nu$ копий $O(U) ; O_{\pi}(U)$ - множество всех $\pi$-симметричных 
на $U$ функций. Очевидно, что $\mathbf{O}(U)$ - модуль над кольцом $O(U)$, а $O_{\pi}(U)$ - подкольцо $O(U)$. Рассматриваем $\mathbf{O}(U)$ как модуль над кольцом $O_{\pi}(U)$.

Совокупность $\left\{O_{\pi}(U)\right\}, U \in \tau_{\pi}$, вместе с гомоморфизмами сужения $\rho_{U, U^{\prime}}$ : $O_{\pi}\left(U^{\prime}\right) \rightarrow O_{\pi}(U), U \subseteq U^{\prime}$, образует предпучок колец над $\left(\mathbb{C}, \tau_{\pi}\right)$. Аналогично, совокупность $\{\mathbf{O}(U)\}, U \in \tau_{\pi}$, вместе с гомоморфизмами сужения $\sigma_{U, U^{\prime}}: \mathbf{O}\left(U^{\prime}\right) \rightarrow$ $\mathbf{O}(U), U \subseteq U^{\prime}$, образует предпучок абелевых групп над ( $\left.\mathbb{C}, \tau_{\pi}\right)[5 ;$ гл. IV, A]. Легко убедиться, что отображение $\sigma_{U, U^{\prime}}, U \subseteq U^{\prime}$, является гомоморфизмом $O_{\pi}\left(U^{\prime}\right)$-модуля $\mathbf{O}\left(U^{\prime}\right)$ в $O_{\pi}(U)$-модуль $\mathbf{O}(U)$, следовательно, предпучок абелевых групп $\{\mathbf{O}(U)\}, U \in \tau_{\pi}$, является предпучком модулей над предпучком колец $\left\{O_{\pi}(U)\right\}$, $U \in \tau_{\pi}$.

Выберем $\lambda \in \Lambda$. Пусть $\widetilde{\lambda}=\pi^{-1}(\lambda)$. Слой предпучка $\{\mathbf{O}(U)\}, U \in \tau_{\pi}$ (индуктивный предел групп $\mathbf{O}(U), \widetilde{\lambda} \subset U \in \tau_{\pi}$, относительно гомоморфизмов $\left.\sigma_{U, U^{\prime}}, U \subseteq U^{\prime}\right)$, обозначим символом $\mathbf{O}(\widetilde{\lambda})$. Аналогично, слой предпучка $\left\{O_{\pi}(U)\right\}, U \in \tau_{\pi}$ (индуктивный предел колец $O_{\pi}(U), \tilde{\lambda} \subset U \in \tau_{\pi}$, относительно гомоморфизмов $\rho_{U, U^{\prime}}$, $\left.U \subseteq U^{\prime}\right)$, обозначим символом $O_{\pi}(\tilde{\lambda})$. Слой $O_{\pi}(\tilde{\lambda})$ обладает структурой кольца, а слой $\mathbf{O}(\tilde{\lambda})$ - структурой модуля над этим кольцом. Элементы $\mathbf{O}(\widetilde{\lambda})$ называются ростками $\nu$-функций, голоморфных в окрестностях $\tilde{\lambda}$, а элементы $O_{\pi}(\widetilde{\lambda})$ - ростками $\pi$-симметричных функций, голоморфных в $\pi$-симметричных окрестностях $\tilde{\lambda}$.

Пусть $I$ - замкнутый подмодуль в $\mathbf{P}$. Обозначим через $I(\widetilde{\lambda})$ минимальный подмодуль $O_{\pi}(\widetilde{\lambda})$-модуля $\mathbf{O}(\widetilde{\lambda})$, включающий $I$. Ясно, что $I(\widetilde{\lambda})$ состоит из всевозможных конечных сумм $\sum c_{i} \varphi^{(i)}$, где $c_{i} \in O_{\pi}(\widetilde{\lambda}), \varphi^{(i)} \in I$. Подмодуль $I(\tilde{\lambda})$ назьвается локальным подмодулем $I$, ассоциированным с $\pi$-слоем $\widetilde{\lambda}$. Говорят, что подмодуль I допускает локальное описание, если справедлива следуюшая импликация:

$$
\varphi \in \mathbf{P}, \quad \varphi \in I(\widetilde{\lambda}) \forall \lambda \in \Lambda \quad \Rightarrow \quad \varphi \in I
$$

Задача локального описания состоит в нахождении условий, при которых замкнутый подмодуль $I \subseteq \mathbf{P}$ допускает локальное описание. Отметим, что импликация (2) была введена впервые И. Ф. Красичковым-Терновским в [6]. Замкнутые подмодули, удовлетворяющие этой импликации, названы им обильнымми.

1.3. Двойственность. Пусть $W$ - замкнутое инвариантное подпространство $\mathbf{H} ; W^{0}$ - аннулятор $W$ в $\mathbf{H}^{*}$. Подпространство An $W:=\mathbf{T}\left(W^{0}\right) \subseteq \mathbf{P}$ обладает структурой замкнутого $\mathbb{C}[\pi]$-подмодуля в $\mathbf{P}$. Соотношение $I=$ An $W$ устанавливает взаимно однозначное соответствие между замкнутыми инвариантными подпространствами $W \subseteq \mathbf{H}$ и замкнутьми $\mathbb{C}[\pi]$-подмодулями $I \subseteq \mathbf{P}$. Подмодуль $I=\operatorname{An} W \subseteq \mathbf{P}$ принято называть аннуляторным подмодулем подпространства $W$.

ТЕОРЕМА ДВОЙСТВЕННОСТИ. Замкнутое инвариантное подпространство $W$ допускает спектральный синтез тогда и только тогда, когда его аннуляторный подмодуль I является обильным.

Эта теорема доказана в [2]. Она вскрывает двойственный характер задач спектрального синтеза и локального описания. Переход от первой задачи ко второй лежит в основе большинства известных работ по спектральному синтезу в комплексной области. 
1.4. Редукция. Обозначим через $\mathbb{C}(\pi)$ поле частных кольца $\mathbb{C}[\pi]$. По известной теореме Люрота о полях рациональных функций $[7 ; \S 7$, теорема 12$] \mathbb{C}(\pi)-$ простое трансцендентное расширение поля $\mathbb{C}$. Другими словами, существует элемент $l \in \mathbb{C}(\pi)$ такой, что $\mathbb{C}(\pi)=\mathbb{C}(l)$, где $\mathbb{C}(l)$ - поле частных кольца $\mathbb{C}[l]$ многочленов от $l$. Всякий элемент $l \in \mathbb{C}(\pi)$, для которого выполняется соотношение $\mathbb{C}(\pi)=\mathbb{C}(l)$, называется әлементом Люрота системы многочленов $\pi_{1}, \ldots, \pi_{q}$. Если $l$ - это элемент Люрота, то и элемент $\frac{a l+b}{c l+d}, a, b, c, d \in \mathbb{C}, a d-b c \neq 0$, является элементом Люрота [7; $\S 7$, теорема 11]. По теореме Нётер [7; $§ 7$, теорема 14] в качестве элемента Люрота может быть выбран многочлен - многочлен Люрота. Если $l$ - многочлен Люрота, то $a l+b, a, b \in \mathbb{C}, a \neq 0,-$ тоже многочлен Люрота. Сушествуют конструктивные процедуры, позволяющие найти многочлен Люрота для любой конкретной системы многочленов (см., например, $[7 ; \S 7])$.

Пусть $I_{0}$ - произвольное множество в $\mathbf{P}$. Обозначим через $I_{0}[\pi]$ множество всевозможных конечных линейных комбинаций элементов $I_{0}$ с коэффициентами из кольца $\mathbb{C}[\pi]$. Множество $I_{0}[\pi]$ является минимальным $\mathbb{C}[\pi]$-подмодулем в $\mathbf{P}$, содержашим множество $I_{0}$. Так как операция умножения на многочлен непрерывна в топологии $\mathbf{P}$, то замыкание $\mathbb{C}[\pi]$-подмодуля $I_{0}[\pi]$ будет также $\mathbb{C}[\pi]$-подмодулем в $\mathbf{P}$. Этот подмодуль будем обозначать $I$ и называть замкнутым $\mathbb{C}[\pi]$-подмодулем в $\mathbf{P}$, порождаемым множеством $I_{0}$. $\mathbb{C}[\pi]$-подмодуль $I$ является минимальным замкнутым $\mathbb{C}[\pi]$-подмодулем в $\mathbf{P}$, содержащим множество $I_{0}$. Аналогичным образом определяется замкнутый $\mathbb{C}[l]-$ подмодуль в $\mathbf{P}$, порожсдаемый множеством $I_{0}$. Этот подмодуль будем обозначать $J$. Здесь $l$ - фиксированный многочлен Люрота системы многочленов $\pi_{1}, \ldots, \pi_{q}$. Если множество $I_{0}$ конечно, то подмодули $I, J$ называются конечно порожденными. Если множество $I_{0}$ состоит из одного элемента, то эти подмодули называются главныци.

Пусть $O_{l}(\widetilde{\xi})$ - кольцо ростков $l$-симметричных функций, голоморфных в $l$-симметричных окрестностях $l$-слоя $\widetilde{\xi} ; \mathbf{O}(\widetilde{\xi})-O_{l}(\widetilde{\xi})$-модуль ростков $\nu$-функций, голоморфных в $l$-симметричных окрестностях $l$-слоя $\widetilde{\xi} ; J(\widetilde{\xi}) \subseteq \mathbf{O}(\widetilde{\xi})-$ локальный подмодуль $J$, ассоциированный с $l$-слоем $\widetilde{\xi}$. Согласно определению замкнутый $\mathbb{C}[l]$-подмодуль $J$ называется обильныцм, если справедлива импликация

$$
f \in \mathbf{P}, \quad f \in J(\widetilde{\xi}) \quad \forall \xi \in \mathbb{C} \quad \Rightarrow \quad f \in J .
$$

В работе [3] доказана следуюшая теорема.

ТЕОРема РЕДУКцИИ. Подмодули I и $Ј$ обильны или нет одновременно.

Для доказательства теоремы редукции принципиальна выполнимость включений

$$
s \mathbb{C}[l] \subseteq \mathbb{C}[\pi] \subseteq \mathbb{C}[l],
$$

из которых вытекают включения

$$
s J \subseteq I \subseteq J .
$$

Здесь $s$ - это некоторый многочлен из кольца $\mathbb{C}[\pi]$. Среди отличных от нуля многочленов $s$, для которых выполняются включения (3), имеется многочлен минимальной степени. Этот многочлен определен однозначно с точностью до постоянного множителя. Отличный от нуля многочлен $s$, для которого выполнены включения (3), имеюший наименьшую из возможных степень и коэффициент при старшем 
члене (относительно $l$ ) равный единице, называется многочленом, ассоциированным с многочленом Люрота l.

Включения (4) отражаются на свойствах локальных подмодулей $I(\widetilde{\lambda}), J(\widetilde{\xi})$. Точнее, всякий $\pi$-слой состоит из конечного множества $l$-слоев. Если $\pi$-слой $\widetilde{\lambda}$ составлен из конечного множества попарно не пересекающихся $l$-слоев $\widetilde{\xi}_{1}, \ldots, \widetilde{\xi}_{k}$, то справедливы следуюшие включения:

$$
s \bigcap_{j=1}^{k} J\left(\widetilde{\xi}_{j}\right) \subseteq I(\widetilde{\lambda}) \subseteq \bigcap_{j=1}^{k} J\left(\widetilde{\xi}_{j}\right)
$$

см. [3; предложение 9].

1.5. Краткое содержание работы. В $\S 2$ доказан критерий обильности, сводящий исследования по спектральному синтезу к проверке конкретных аналитико-алгебраических свойств замкнутых $\mathbb{C}[\pi]$-подмодулей в $\mathbf{P}$; рассмотрены ситуации, в которых эта проверка может быть упрощена. В $\S 3$ замкнутые подпространства в Н описаны в терминах однородных сверточных уравнений. Здесь же вскрывается строение аннуляторных подмодулей для подпространств решений систем однородных сверточных уравнений. Этим открывается путь к применению результатов по локальному описанию в частных ситуациях. Основные следствия критерия обильности и теоремы редукции приведены в $\S 4$.

\section{§2. Критерий обильности}

Теорема редукции сводит задачу локального описания замкнутых подмодулей в $\mathbf{P}$ над кольцом многочленов, порожденньг совокупностью многочленов $\pi_{1}, \ldots$, $\pi_{q}$, к задаче локального описания замкнутых подмодулей в $\mathbf{P}$ над кольцом многочленов, порожденным одним многочленом $l$. Последняя задача исследовалась ранее. Практически все известные результаты по локальному описанию замкнутых $\mathbb{C}[l]$-подмодулей в $\mathbf{P}$ связаны с ограничением: $\Omega_{1}, \ldots, \Omega_{\nu}-$ выпуклые области в $\mathbb{C}$. Это объясняется тем, что все известные исследования замкнутых подмодулей в $\mathbf{P}$ опираются на предположение, что в модуле $\mathbf{P}$ выполнены следуюшие аксиомы.

Аксиома сходимости. Топология $\mathbf{P}$ мажсорирует топологию поточечной сходимости.

АКСИОМА РАВНОМЕРНОЙ УСТОЙЧИВОСТИ. Для любой окрестности нуля $V \subseteq \mathbf{P}$ существует окрестность нуля $U \subseteq \mathbf{P}$ такая, что справедлива импликащия

$$
\varphi \in U, \quad(\zeta-a) \mid \varphi \quad \Rightarrow \quad \frac{\varphi(\zeta)}{\zeta-a} \in V
$$

Здесь $(\zeta-a) \mid \varphi$ означает, что двучлен $\zeta-a$ делит $\varphi=\left(\varphi_{1}, \ldots, \varphi_{\nu}\right)$, т.е. $\frac{\varphi_{j}(\zeta)}{\zeta-a}$ голоморфины в окрестности точки $a$.

Эти аксиомы впервые сформулированы в работе И. Ф. Красичкова-Терновского [6].

Выполнимость аксиомы сходимости не связана с условием выпуклости областей $\Omega_{1}, \ldots, \Omega_{\nu}$. Эта аксиома вьполняется, если $\Omega_{1}, \ldots, \Omega_{\nu}$ - односвязные области [8]. 
В то же время, для выполнимости аксиомы равномерной устойчивости выпуклость областей $\Omega_{1}, \ldots, \Omega_{\nu}$ является принципиальной [8].

Согласно известному критерию И. $Ф$. Красичкова-Терновского проверка обильности замкнутого $\mathbb{C}[l]$-подмодуля в $\mathbf{P}$ сводится к проверке его устойчивости и насыщенности (случай $l(\zeta)=\zeta$ изучен в [6], [9]; общий случай рассмотрен в [10], [11]; ситуация $l(\zeta)=\zeta^{m}$ исследована автором [12], [13]).

2.1. Устойчивые подмодули. Пусть $\Omega_{1}, \ldots, \Omega_{\nu}-$ выпуклые области в $\mathbb{C}$. $\mathbb{C}[\pi]$-подмодуль $I \subseteq \mathbf{P}$ назьвается устойчивым, если для любого $r \in \mathbb{C}[\pi]$ справедлива импликация

$$
f \in I, \quad \frac{f}{r} \in I(\tilde{\lambda}) \quad \forall \lambda \in \Lambda \quad \Rightarrow \quad \frac{f}{r} \in I .
$$

Обозначим через $l$ фиксированный многочлен Люрота системы многочленов $\pi_{1}, \ldots, \pi_{q}$; и пусть $s$ - многочлен, ассоциированный с многочленом Люрота $l ; I_{0}-$ произвольное множество в $\mathbf{P} ; I$ - замкнутьй $\mathbb{C}[\pi]$-подмодуль в $\mathbf{P}$, порождаемый множеством $I_{0} ; J$ - замкнутьй $\mathbb{C}[l]$-подмодуль в $\mathbf{P}$, порождаемый множеством $I_{0}$ (см. п. 1.4). Для $\mathbb{C}[l]$-подмодуля $J$ импликация (5) эквивалентна импликации

$$
f \in J, \quad \frac{f}{l-l(\xi)} \in J(\widetilde{\xi}) \quad \Rightarrow \quad \frac{f}{l-l(\xi)} \in J,
$$

где $\xi$ - фиксированное комплексное число, $\widetilde{\xi}=l^{-1}(\xi)$. Выполнимость этой импликации не зависит от выбора числа $\xi[10$; предложение 3.1$]$.

ТЕОРемА 2.1. Подмодули I и Ј устойчивъ или нет одновременно.

ДокАЗАТЕЛЬСТво. Предположим, что $\mathbb{C}[\pi]$-подмодуль $I$ является устойчивым. Значит, для любого многочлена $r \in \mathbb{C}[\pi]$ справедлива импликация (5). Нужно доказать, что условие $f \in J, \frac{f}{l-l(\xi)} \in J(\widetilde{\xi})$ влечет включение $\frac{f}{l-l(\xi)} \in J$. В силу [3; лемма 7] это условие влечет сушествование элемента $\psi \in I_{0}[l]$ такого, что $\frac{f}{l-l(\xi)}=\psi+s g$, где $g \in \mathbf{P}, g \in J(\widetilde{\xi}) \forall \xi \in \mathbb{C}$. Согласно [3; предложение 9] $s g \in I(\widetilde{\lambda}) \forall \lambda \in \Lambda$. При этом

$$
s g=\frac{s f-s(l-l(\xi)) \psi}{s(l-l(\xi))}, \quad s f \in s J \subset I, \quad s(l-l(\xi)) \psi \in I_{0}[\pi] \subset I .
$$

В силу импликации (5) $s g \in I$. Значит, $\frac{f}{l-l(\xi)}=\psi+s g \in J$. Следовательно, $\mathbb{C}[l]$-подмодуль $J$ является устойчивым.

Обратно, предположим, что $\mathbb{C}[l]$-подмодуль $J$ является устойчивым. Выберем произвольный многочлен $r \in \mathbb{C}[\pi]$. Нужно доказать, что справедлива импликация (5). Пусть $f \in I, f / r \in I(\widetilde{\lambda}) \forall \lambda \in \Lambda$. В силу [3; лемма 8] это условие влечет сушествование элемента $\psi \in I_{0}[\pi]$ такого, что $f / r=\psi+s g$, где $g \in \mathbf{P}$, $g \in J(\widetilde{\xi}) \forall \xi \in \mathbb{C}$. Значит, $(f-r \psi) /(s r) \in J(\widetilde{\xi}) \forall \xi \in \mathbb{C}$. При этом $f \in I \subset J$, $r \psi \in I_{0}[\pi] \subset I \subseteq J$, т.е. $f-r \psi \in J$. Устойчивость подмодуля $J$ влечет включение $g=(f-r \psi) /(s r) \in J$. Значит, $f / r=\psi+s g \in I$. Теорема доказана. 
СледСтвиЕ 2.1. Замыкание устойчивого $\mathbb{C}[\pi]$-подмодуля в $\mathbf{P}$ является устойчивым $\mathbb{C}[\pi]$-подмодулем в $\mathbf{P}$.

ДокАЗАТЕЛЬСТво. Пусть $I_{0}-$ устойчивый $\mathbb{C}[\pi]$-подмодуль в $\mathbf{P}, I$ - замыкание $I_{0}$ в топологии $\mathbf{P}$. Покажем, что подмодуль $I$ является устойчивым. Обозначим через $I_{0}[l]$ минимальный $\mathbb{C}[l]$-подмодуль в $\mathbf{P}$, включающий $I_{0}$, а через $J$ - замькание $I_{0}[l]$ в топологии $\mathbf{P}$. Прежде всего убедимся, что $\mathbb{C}[l]$-подмодуль $I_{0}[l]$ является устойчивьм. Для этого нужно доказать, что условие $f \in I_{0}[l], \frac{f}{l-l(\xi)} \in$ $I_{0}[l](\widetilde{\xi})$ влечет включение $\frac{f}{l-l(\xi)} \in I_{0}[l]$. Так как $I_{0}[l] \subseteq J$, то $I_{0}[l](\widetilde{\xi}) \subseteq J(\widetilde{\xi})$ $\forall \xi \in \mathbb{C}$. Значит, $\frac{f}{l-l(\xi)} \in J(\widetilde{\xi}) \forall \xi \in \mathbb{C}$. В силу [3; лемма 7] сушествует элемент $\psi \in I_{0}[l]$ такой, что $\frac{f}{l-l(\underbrace{}_{\tilde{\lambda})}}=\psi+s g$, где $g \in \mathbf{P}, g \in J(\widetilde{\xi}) \forall \xi \in \mathbb{C}$. Согласно [3; предложение 9] $s g \in I(\widetilde{\lambda}) \forall \lambda \in \Lambda$, а ввиду [3; лемма 5] $I(\widetilde{\lambda})=I_{0}(\widetilde{\lambda}) \forall \lambda \in \Lambda$. Значит, $s g \in I_{0}(\tilde{\lambda}) \forall \lambda \in \Lambda$. При этом

$$
s g=\frac{s f-s(l-l(\xi)) \psi}{s(l-l(\xi))}, \quad s f \in s I_{0}[l] \subset I_{0}[\pi]=I_{0}, \quad s(l-l(\xi)) \psi \in I_{0}[\pi]=I_{0} .
$$

В силу импликации (5) $s g \in I_{0}$. Значит, $\frac{f}{l-l(\xi)}=\psi+s g \in I_{0}[l]$. Таким образом, устойчивость $\mathbb{C}[l]$-подмодуля $I_{0}[l]$ доказана. В силу $[10 ;$ предложение 3.2$]$ $\mathbb{C}[l]$-подмодуль $J$ является устойчивым. Осталось сослаться на теорему 2.1 и заключить, что $\mathbb{C}[\pi]$-подмодуль $I$ является устойчивым.

Пусть $\widetilde{\lambda}-\pi$-слой. Система элементов $u^{(1)}, \ldots, u^{(k)} \in \mathbf{O}(\widetilde{\lambda})$ называется независимой над кольцом $O_{\pi}(\widetilde{\lambda})$, если справедлива импликация

$$
c_{1} u^{(1)}+\cdots+c_{k} u^{(k)}=0, \quad c_{i} \in O_{\pi}(\widetilde{\lambda}) \quad \Rightarrow \quad c_{i}=0, \quad i=1, \ldots, k .
$$

В противном случае система $u^{(1)}, \ldots, u^{(k)}$ называется зависимой над кольцом $O_{\pi}(\widetilde{\lambda})$. Система элементов $\varphi^{(1)}, \ldots, \varphi^{(k)} \in \mathbf{P} \pi$-независима, если эта система независима над любьм кольцом $O_{\pi}(\widetilde{\lambda}), \lambda \in \Lambda$. В противном случае система $\varphi^{(1)}, \ldots, \varphi^{(k)}$ называется $\pi$-зависимой.

ПРЕДЛОЖЕНИЕ 2.2. Если систем $\underset{\sim}{(}$ әлементов $\varphi^{(1)}, \ldots, \varphi^{(k)} \in \mathbf{P}$ зависима над фиксированным кольцом $O_{\pi}\left(\widetilde{\lambda}_{0}\right)$, то она зависима над любым другим кольцом $O_{\pi}(\widetilde{\lambda}), \lambda \in \Lambda$.

Доказательство. Пусть система элементов $\varphi^{(1)}, \ldots, \varphi^{(k)} \in \mathbf{P}$ зависима над кольцом $O_{\pi}\left(\widetilde{\lambda}_{0}\right)$. Значит, найдется открытая $\pi$-симметричная окрестность $U_{0}$ $\pi$-слоя $\widetilde{\lambda}_{0}$, на которой выполняется тождество $c_{1} \varphi^{(1)}+\cdots+c_{k} \varphi^{(k)} \equiv 0$, где коэффициенты $c_{1}, \ldots, c_{k}$ принадлежат кольцу $O_{\pi}\left(U_{0}\right)$ и не все из них являются тождественными нулями. Найдется точка $\zeta^{\prime} \in U_{0}$, в которой многочлен $s$ и хотя бы один из коэффициентов $c_{1}, \ldots, c_{k}$ отличны от нуля. Выберем открытую $l$-симметричную окрестность $U \subseteq U_{0} \quad l$-слоя $\widetilde{\xi}^{\prime}=l^{-1}\left(l\left(\zeta^{\prime}\right)\right)$, лежащую вне нулевого 
множества многочлена $s$. Согласно [3; предложение 4] множество $U$ является $\pi$-симметричным. При этом $c_{1}, \ldots, c_{k} \in O_{\pi}(U)$. Из [3; предложение 5] имеем $O_{\pi}(U)=O_{l}(U)$, значит, $c_{1}, \ldots, c_{k} \in O_{l}(U)$. Следовательно, система $\varphi^{(1)}, \ldots, \varphi^{(k)}$ зависима над кольцом $O_{l}\left(\widetilde{\xi}^{\prime}\right)$. В силу [14; предложение 3.2 , следствие 2] эта система зависима над любым кольцом $O_{l}(\widetilde{\xi}), \widetilde{\xi} \subset \mathbb{C}$. Выберем произвольный $\pi$-слой $\widetilde{\lambda}$. Он состоит из конечной совокупности $l$-слоев $\widetilde{\xi}_{1}, \ldots, \widetilde{\xi}_{m}$. Система $\varphi^{(1)}, \ldots, \varphi^{(k)}$ зависима над каждым кольцом $O_{l}\left(\widetilde{\xi}_{1}\right), \ldots, O_{l}\left(\widetilde{\xi}_{m}\right)$. Значит, найдутся попарно не пересекающиеся открытые $l$-симметричные множества $U_{1} \supset \widetilde{\xi}_{1}, \ldots, U_{m} \supset \widetilde{\xi}_{m}$, на которых выполняются тождества

$$
c_{1}^{(1)} \varphi^{(1)}+\cdots+c_{k}^{(1)} \varphi^{(k)} \equiv 0, \quad \ldots, \quad c_{1}^{(m)} \varphi^{(1)}+\cdots+c_{k}^{(m)} \varphi^{(k)} \equiv 0 .
$$

Здесь $c_{1}^{(j)}, \ldots, c_{k}^{(j)} \in O_{l}\left(U_{j}\right)$ и в каждой совокупности $c_{1}^{(j)}, \ldots, c_{k}^{(j)}$ имеется элемент, отличный от тождественного нуля. Определим на открытом $l$-симметричном множестве $U=U_{1} \cup \cdots \cup U_{m} l$-симметричные функции $c_{1}, \ldots, c_{k}$, полагая $c_{1}(\zeta)=$ $c_{1}^{(j)}(\zeta), \ldots, c_{k}(\zeta)=c_{k}^{(j)}(\zeta)$ для $\zeta \in U_{j}$. Замечаем, что на $U$ выполняется тождество $c_{1} \varphi^{(1)}+\cdots+c_{k} \varphi^{(k)} \equiv 0$. Множество $U$ содержит $\pi$-слой $\tilde{\lambda}$. Значит, найдется открытое $\pi$-симметричное множество $V$ такое, что $\tilde{\lambda} \subset V \subseteq U[3 ; \S 1$, п. 1.2]. Функции $c_{1}, \ldots, c_{k}$ принадлежат кольцу $O_{l}(V)$. Значит, $s c_{1}, \ldots, s c_{k} \in O_{\pi}(V)[3$; предложение 6]. При этом на $V$ выполняется тождество $s c_{1} \varphi^{(1)}+\cdots+s c_{k} \varphi^{(k)} \equiv 0$. Следовательно, система $\varphi^{(1)}, \ldots, \varphi^{(k)}$ зависима над кольцом $O_{\pi}(\widetilde{\lambda})$. Предложение доказано.

Рассмотрим некоторые следствия предложения 2.2 .

СлеДСТВИЕ 2.1. Система әлементов $\varphi^{(1)}, \ldots, \varphi^{(k)} \in \mathbf{P} \pi$-независима тогда и только тогда, когда она l-независима.

ДокАЗАТЕЛЬство. Выберем произвольњый $\pi$-слой $\widetilde{\lambda}$, не пересекающийся с нулевьп множеством многочлена $s$. Он совпадает с $l$-слоем $l^{-1}(l(\zeta)), \zeta \in \widetilde{\lambda}[3$; предложение 3]. Из предложения 2.2 вытекает, что $\pi$-независимость системы элементов $\varphi^{(1)}, \ldots, \varphi^{(k)} \in \mathbf{P}$ эквивалентна ее независимости над кольцом $O_{\pi}(\widetilde{\lambda})$, а $l$-независимость этой системы эквивалентна ее независимости над кольцом $O_{l}(\tilde{\lambda})$. Но из [3; предложение 5$]$ следует, что эти кольца совпадают. Значит, $\pi$-независимость и $l$-независимость системы $\varphi^{(1)}, \ldots, \varphi^{(k)}$ эквивалентны.

Следуя И.Ф. Красичкову-Терновскому [6], максимальное число элементов в $\pi$-независимых системах из множества $I_{0} \subseteq \mathbf{P}$ называем $\pi$-рангом множества $I_{0}$ и обозначаем $\pi-\operatorname{Rank} I_{0}$.

СЛЕДСТВИЕ 2.2. Для любого множества $I_{0} \subseteq \mathbf{P}$ выполняются соотношения

$$
\pi-\operatorname{Rank} I_{0}=l-\operatorname{Rank} I_{0} \leqslant \nu m,
$$

где $m$ - степень многочлена l.

ДокаЗАтельство. Равенство $\pi$-Rank $I_{0}=l$-Rank $I_{0}$ вытекает из следствия 2.1. Неравенство $l$-Rank $I_{0} \leqslant \nu m$ выполняется в силу [14; предложение 3.2 , следствие 1]. 
СлеДСтВИЕ 2.3. Пусть $I$ - замкнутый $\mathbb{C}[\pi]-$ подмодуль в $\mathbf{P}$, порожсденный множсеством $I_{0} \subseteq \mathbf{P}, J$ - замкнутый $\mathbb{C}[l]-$ подмодуль в $\mathbf{P}$, порожсденный множеством $I_{0}$. Тогда

$$
\pi-\operatorname{Rank} I=l-\operatorname{Rank} J=l-\operatorname{Rank} I_{0} .
$$

ДокаЗАтЕльство. Неравенство $\pi$-Rank $I \leqslant l$-Rank $J$ вытекает из следствия 2.1 и вложения $I \subseteq J$ (см. (4)). Докажем выполнимость обратного неравенства. Пусть $\varphi^{(1)}, \ldots, \varphi^{(k)}-l$-независимая система элементов из $J$. Из левого включения в $(4)$ вытекает, что $s \varphi^{(1)}, \ldots, s \varphi^{(k)}-l$-независимая система элементов из $I$. По следствию 2.1 система $s \varphi^{(1)}, \ldots, s \varphi^{(k)}$ является $\pi$-независимой. Таким образом, $l$-Rank $J$ не может быть больше $\pi-\operatorname{Rank} I$ и вьполнимость равенства $\pi$-Rank $I=l$-Rank $J$ доказана. С другой стороны, в силу [3; лемма 6] локальные подмодули $J(\widetilde{\xi})$ и $I_{0}(\widetilde{\xi})$, ассоциированные с произвольным $l$-слоем $\widetilde{\xi}$, совпадают. Отсюда $[14 ;$ предложение 3.3] следует равенство $l$-Rank $J=l-\operatorname{Rank} I_{0}$. Следствие доказано.

ПРЕДЛОЖЕНИЕ 2.3. Замкнутый $\mathbb{C}[\pi]$-подмодуль $I$, порожсденный $\pi$-независимой системой $\varphi^{(1)}, \ldots, \varphi^{(k)} \in \mathbf{P}$, является устойчивьм, в частности каждый главный $\mathbb{C}[\pi]-$ подмодуль в $\mathbf{P}$ является устойчивъцм.

ДокАЗАТЕЛЬство. Пусть $\varphi^{(1)}, \ldots, \varphi^{(k)}-\pi$-независимая система элементов из $\mathbf{P}$, и пусть $I_{0}=\left\{\varphi^{(1)}, \ldots, \varphi^{(k)}\right\}, I-$ замкнутый $\mathbb{C}[\pi]$-подмодуль в $\mathbf{P}$, порожденный множеством $I_{0}, J$ - замкнутый $\mathbb{C}[l]$-подмодуль в $\mathbf{P}$, порожденный множеством $I_{0}$. По следствию 2.1 система элементов $\varphi^{(1)}, \ldots, \varphi^{(k)}$ является $l$-независимой. Значит, $\mathbb{C}[l]$-подмодуль $J$ является устойчивым $[10$; предложение 3.3$]$. Следовательно, по теореме $2.1 \mathbb{C}[\pi]$-подмодуль $I$ является устойчивым.

Далее нам потребуется понятие $l$-индекса множества $I_{0} \subseteq \mathbf{P}\left[14 ;\right.$ п. 3.3]. Пусть $I_{0}$ - произвольное множество в $\mathbf{P} \pi$-ранга $k ; \varphi^{(1)}, \ldots, \varphi^{(k)}-\pi$-независимая система элементов из $I_{0} ; m$ - степень многочлена $l$. Согласно [14; предложение 3.1] элемент $\varphi^{(n)}, n=1, \ldots, k$, представляется единственным образом в виде

$$
\varphi^{(n)}(\zeta)=\sum_{i=0}^{m-1} \zeta^{i} \psi_{i}^{(n)}(l(\zeta)), \quad \psi_{i}^{(n)}=\left(\psi_{i, 1}^{(n)}, \ldots, \psi_{i, \nu}^{(n)}\right) \in O^{\nu}(\mathbb{C})
$$

Обозначим через $\left(\varphi^{(1)}, \ldots, \varphi^{(k)}\right)$ матрицу размера $m \nu \times k$, составленную из $k$ столбцов, состоящих из $\psi_{0,1}^{(n)}, \ldots, \psi_{0, \nu}^{(n)}, \ldots, \psi_{m-1,1}^{(n)}, \ldots, \psi_{m-1, \nu}^{(n)}$ соответственно. Минор $k$-го порядка матрищы $\left(\varphi^{(1)}, \ldots, \varphi^{(k)}\right)$, составленньй из строк с номерами $i_{1}, \ldots, i_{k}, 1 \leqslant i_{1}<\cdots<i_{k} \leqslant m \nu$, обозначается

$$
\left[\begin{array}{ccc}
\varphi^{(1)} & \ldots & \varphi^{(k)} \\
i_{1} & \ldots & i_{k}
\end{array}\right]
$$

Выберем произвольньй $l$-слой $\widetilde{\xi}=l^{-1}(l(\xi)) ; l$-индекс $l$-Ind $\operatorname{In}_{\tilde{\xi}}$ множества $I_{0} \subseteq \mathbf{P}$ $\pi$-ранга $k$ в точке $\omega \in \widetilde{\xi}$ есть минимальная кратность нуля в точке $\xi$ определителей (6) по всевозможным $\pi$-независимым системам $\varphi^{(1)}, \ldots, \varphi^{(k)} \in I_{0}$ и всевозможным наборам $i_{1}, \ldots, i_{k}, 1 \leqslant i_{1}<\cdots<i_{k} \leqslant m \nu$. 
Рассмотрим конечную совокупность $I^{(1)}, \ldots, I^{(k)}$ замкнутых $\mathbb{C}[\pi]$-подмодулей в $\mathbf{P}$ и обозначим через $I$ замькание в топологии $\mathbf{P}$ множества элементов вида $\varphi^{(1)}+\cdots+\varphi^{(k)}, \varphi^{(i)} \in I^{(i)}$. Множество $I$ является замкнутьм $\mathbb{C}[\pi]$-подмодулем в $\mathbf{P}$ и назьвается суммой $\mathbb{C}[\pi]$-подмодулей $I^{(1)}, \ldots, I^{(k)}[15]$. Легко проверить, что подмодуль $I$ совпадает с замкнутым $\mathbb{C}[\pi]$-подмодулем в $\mathbf{P}$, порождаемым множеством $I_{0}=I^{(1)} \cup \cdots \cup I^{(k)}$ (см. п. 1.4). Если $\mathbb{C}[\pi]$-подмодули $I^{(1)}, \ldots, I^{(k)}-$ главные с образующими $\varphi^{(1)}, \ldots, \varphi^{(k)}$ соответственно, то подмодуль $I-$ конечно порожденньй $\mathbb{C}[\pi]$-подмодуль, порождаемый множеством $\left\{\varphi^{(1)}, \ldots, \varphi^{(k)}\right\}$. Устойчивость сумм $\mathbb{C}[l]$-подмодулей исследована И. $\Phi$. Красичковым-Терновским в [10]. Два следующих предложения являются аналогами предложений 3.4 и 3.5 из [10].

Зафиксируем простой $\pi$-слой, не пересекаюшийся с нулевым множеством многочлена $s$. В силу [3; предложение 3$]$ фиксированный $\pi$-слой является и $l$-слоем. Обозначим его через $\widetilde{\xi}$. Функция $\alpha^{(i)}: \widetilde{\xi} \rightarrow \mathbb{C}^{\nu}$ называется допустимой относительно подмодуля $I^{(i)} \subseteq \mathbf{P}$, если сушествует элемент $\varphi \in I^{(i)}$ такой, что $\alpha^{(i)}(\omega)=\varphi(\omega)$ $\forall \omega \in \widetilde{\xi}$. Рассмотрим набор $\alpha=\left\{\alpha^{(1)}, \ldots, \alpha^{(k)}\right\}$ функций $\widetilde{\xi} \rightarrow \mathbb{C}^{\nu}$, допустимых относительно $\mathbb{C}[\pi]$-подмодулей $I^{(1)}, \ldots, I^{(k)}$ соответственно. Положим

$$
\begin{aligned}
T(I, \alpha, \widetilde{\xi})=\{\varphi & =\varphi^{(1)}+\cdots+\varphi^{(k)}: \varphi^{(i)} \in I^{(i)} \\
& \left.\varphi^{(i)}(\omega)=\alpha^{(i)}(\omega) \forall \omega \in \widetilde{\xi}, i=1, \ldots, k\right\} .
\end{aligned}
$$

В предположении, что $\mathbb{C}[\pi]$-подмодули $I^{(1)}, \ldots, I^{(k)}$ являются устойчивыми, оказывается справедливьм следуюшее предложение.

ПРЕДЛОЖЕНИЕ 2.4. Пусть

$$
l-\operatorname{Ind}_{\widetilde{\xi}} I^{(1)}=\cdots=l-\operatorname{Ind}_{\widetilde{\xi}} I^{(k)}=0 .
$$

Для того чтобы подмодуль I был устойчив, необходимо и достаточно, чтобы выполнялось условие: для любого набора $\alpha=\left\{\alpha^{(1)}, \ldots, \alpha^{(k)}\right\}, \alpha^{(1)}+\cdots+$ $\alpha^{(k)}=0$, функицй, допустимых относительно $I^{(1)}, \ldots, I^{(k)}$ соответственно, замыкание множества $T(I, \alpha, \widetilde{\xi})$ в топологии $\mathbf{P}$ содержит нулевой әлемент.

ДокАЗАтЕльство. Необходимость. Пусть $I$ - сумма $\mathbb{C}[\pi]$-подмодулей $I^{(1)}, \ldots$, $I^{(k)} ; \widehat{I}^{(1)}, \ldots, \widehat{I}^{(k)}$ - замкнутые $\mathbb{C}[l]$-подмодули в $\mathbf{P}$, порождаемые $\mathbb{C}[\pi]$-подмодулями $I^{(1)}, \ldots, I^{(k)}$ соответственно. Считаем, что подмодуль $I$ является устойчивым. Обозначим через $J$ сумму $\mathbb{C}[l]$-подмодулей $\widehat{I}^{(1)}, \ldots, \widehat{I}^{(k)}$. Как уже отмечалось, замкнутый $\mathbb{C}[\pi]$-подмодуль в $\mathbf{P}$, порождаемый множеством $I_{0}=I^{(1)} \cup \cdots \cup I^{(k)}$, совпадает с $I$. С другой стороны, замкнутый $\mathbb{C}[l]$-подмодуль в $\mathbf{P}$, порождаемый множеством $I_{0}$, содержится в $J$ и, в то же время, включает множество $\widehat{I}_{0}=\widehat{I}^{(1)} \cup$ $\cdots \cup \widehat{I}^{(k)}$. Это означает, что он совпадает с $J$. Таким образом, замкнутые подмодули $I$ и $J$ порождаются одним и тем же множеством $I_{0}$. По теореме 2.1 устойчивость подмодуля $I$ влечет устойчивость подмодуля $J$. Выберем произвольньй набор $\alpha=\left\{\alpha^{(1)}, \ldots, \alpha^{(k)}\right\}, \alpha^{(1)}+\cdots+\alpha^{(k)}=0$, функций, допустимых относительно подмодулей $I^{(1)}, \ldots, I^{(k)}$ соответственно. Из включений $I^{(1)} \subseteq \widehat{I}^{(1)}, \ldots, I^{(k)} \subseteq \widehat{I}^{(k)}$ вытекает, что функции $\alpha^{(1)}, \ldots, \alpha^{(k)}$ являются допустимыми относительно подмодулей $\widehat{I}^{(1)}, \ldots, \widehat{I}^{(k)}$ соответственно и при этом

$$
l-\operatorname{Ind}_{\widetilde{\xi}} \widehat{I}^{(i)}=l-\operatorname{Ind}_{\widetilde{\xi}} I^{(i)}=0, \quad i=1, \ldots, k .
$$


В силу [10; предложение 3.4$]$ замькание множества $T(J, \alpha, \widetilde{\xi})$ в топологии $\mathbf{P}$ содержит нулевой элемент. Из непрерывности в $\mathbf{P}$ операции умножения на многочлен следует, что тем же свойством обладает множество $\frac{s}{s(\widetilde{\xi})} T(J, \alpha, \widetilde{\xi})$. Левое включение в (4) влечет включения $s \widehat{I}^{(1)} \subseteq I^{(1)}, \ldots, s \widehat{I}^{(k)} \subseteq I^{(k)}$. Значит, последнее множество является частью множества $T(I, \alpha, \widetilde{\xi})$. Следовательно, замькание множества $T(I, \alpha, \widetilde{\xi})$ в топологии $\mathbf{P}$ содержит нулевой элемент. Необходимость доказана.

Достаточность. Предположим, что для любого набора $\alpha=\left\{\alpha^{(1)}, \ldots, \alpha^{(k)}\right\}$, $\alpha^{(1)}+\cdots+\alpha^{(k)}=0$, функций, допустимых относительно подмодулей $I^{(1)}, \ldots, I^{(k)}$ соответственно, замыкание множества $T(I, \alpha, \widetilde{\xi})$ в топологии $\mathbf{P}$ содержит нулевой элемент. Выберем произвольный набор $\alpha=\left\{\alpha^{(1)}, \ldots, \alpha^{(k)}\right\}, \alpha^{(1)}+\cdots+$ $\alpha^{(k)}=0$, функций, допустимых относительно подмодулей $\widehat{I}^{(1)}, \ldots, \widehat{I}^{(k)}$ соответственно. По определению набора $\alpha$ существуют $\varphi^{(1)} \in \widehat{I}^{(1)}, \ldots, \varphi^{(k)} \in \widehat{I}^{(k)}$ такие, что $\alpha^{(1)}(\omega)=\varphi^{(1)}(\omega), \ldots, \alpha^{(k)}(\omega)=\varphi^{(k)}(\omega) \forall \omega \in \widetilde{\xi}$. При этом

$$
\begin{gathered}
\alpha^{(1)}(\omega)=\frac{s(\omega)}{s(\widetilde{\xi})} \varphi^{(1)}(\omega), \ldots, \alpha^{(k)}(\omega)=\frac{s(\omega)}{s(\widetilde{\xi})} \varphi^{(k)}(\omega) \quad \forall \omega \in \widetilde{\xi}, \\
\frac{s}{s(\widetilde{\xi})} \varphi^{(1)} \in I^{(1)}, \ldots, \frac{s}{s(\widetilde{\xi})} \varphi^{(k)} \in I^{(k)} .
\end{gathered}
$$

Следовательно, функции $\alpha^{(1)}, \ldots, \alpha^{(k)}$ являются допустимыми относительно подмодулей $I^{(1)}, \ldots, I^{(k)}$ соответственно. По предположению замькание множества $T(I, \alpha, \widetilde{\xi})$ в топологии $\mathbf{P}$ содержит нулевой элемент. Но множество $T(I, \alpha, \widetilde{\xi})$ является частью множества $T(J, \alpha, \widetilde{\xi})$. Значит, замыкание множества $T(J, \alpha, \widetilde{\xi})$ в топологии $\mathbf{P}$ содержит нулевой элемент. Согласно [10; предложение 3.4$]$ подмодуль $J$ является устойчивым. По теореме 2.1 таковьм же является подмодуль $I$. Предложение доказано.

Предположим, что в условиях предыдушего предложения замкнутые $\mathbb{C}[\pi]$-подмодули $I^{(1)}, \ldots, I^{(k)}$ - главные $\mathbb{C}[\pi]$-подмодули в $\mathbf{P}$, порождаемые $\varphi^{(1)}, \ldots, \varphi^{(k)}$ соответственно. Положим

$$
A(\widetilde{\xi})=\left\{a=\left(a_{1}, \ldots, a_{k}\right) \in \mathbb{C}^{k}: a_{1} \varphi^{(1)}(\omega)+\cdots+a_{k} \varphi^{(k)}(\omega)=0 \forall \omega \in \widetilde{\xi}\right\} .
$$

ПРЕДЛОЖЕНИЕ 2.5. Для того чтобы подмодуль I был устойчивым, необходимо и достаточно, чтобь для каждого вектора а $\in A(\widetilde{\xi})$ существовали обобщенные последовательности $\left\{p_{1 \sigma}\right\}, \ldots,\left\{p_{k \sigma}\right\}, \sigma \in \Sigma$, многочленов из кольиа $\mathbb{C}[l]$ такие, что:

1) $p_{1 \sigma}(\widetilde{\xi})=a_{1}, \ldots, p_{k \sigma}(\widetilde{\xi})=a_{k}$;

2) $p_{1 \sigma} \varphi^{(1)}+\cdots+p_{k \sigma} \varphi^{(k)} \rightarrow 0$ в mопологии $\mathbf{P}$.

ДокАЗАТЕЛЬСтво. Пусть $\widehat{I}^{(1)}, \ldots, \widehat{I}^{(k)}-\mathbb{C}[l]$-подмодули в $\mathbf{P}$, порождаемые подмодулями $I^{(1)}, \ldots, I^{(k)}$ соответственно. Так как подмодули $I^{(1)}, \ldots, I^{(k)}-$ главные $\mathbb{C}[\pi]$-подмодули в $\mathbf{P}$, порождаемые элементами $\varphi^{(1)}, \ldots, \varphi^{(k)}$ соответственно, то $\widehat{I}^{(1)}, \ldots, \widehat{I}^{(k)}$ - главные $\mathbb{C}[l]$-подмодули в $\mathbf{P}$, порождаемые теми же элементами. Пусть $J$ - сумма $\mathbb{C}[l]$-подмодулей $\widehat{I}^{(1)}, \ldots, \widehat{I}^{(k)}$. Как уже отмечалось при доказательстве предложения 2.4 , подмодуль $I$ совпадает с замкнутым 
$\mathbb{C}[\pi]$-подмодулем в $\mathbf{P}$, порождаемым множеством $I^{(1)} \cup \cdots \cup I^{(k)}$, а подмодуль $J$ совпадает с замкнутым $\mathbb{C}[l]$-подмодулем в $\mathbf{P}$, порождаемьм тем же множеством. По теореме 2.1 устойчивость подмодуля $I$ эквивалентна устойчивости подмодуля $J$. С другой стороны, в силу [10; предложение 3.5$]$ устойчивость подмодуля $J$ эквивалентна сушествованию обобщенных последовательностей $\left\{p_{1 \sigma}\right\}, \ldots,\left\{p_{k \sigma}\right\}$, $\sigma \in \Sigma$, многочленов из кольца $\mathbb{C}[l]$, удовлетворяющих условиям 1) и 2), для каждого вектора $a \in A(\widetilde{\xi})$. Тем самым предложение доказано.

2.2. Насыщенные подмодули. Пусть $I-\mathbb{C}[\pi]$-подмодуль в $\mathbf{P}$ ранга $k, \varphi \in \mathbf{P}$. Рассмотрим локальное представление

$$
\varphi=\sum_{i=1}^{k} c_{i} u^{(i)}
$$

в точке $\zeta$. Здесь $u^{(1)}, \ldots, u^{(k)} \in I, c_{1}, \ldots, c_{k} \in O_{\pi}(\widetilde{\lambda}), \widetilde{\lambda}-\pi$-слой, содержащий $\zeta$. Локальное представление такого типа будем называть $\pi$-представлением. Пусть $\rho$ - непрерывная полунорма на Р. Представление (7) называется $\rho$-допустимымм, если $\rho\left(u^{(1)}\right) \leqslant 1, \ldots, \rho\left(u^{(k)}\right) \leqslant 1$. Далее, обозначим через $c(\zeta)$ значение ростка $c \in O_{\pi}(\widetilde{\lambda})$ в точке $\zeta$. Введем функцию

$$
\rho_{I}(\zeta, \varphi)=\inf \left\{1+\sum\left|c_{i}(\zeta)\right|\right\}
$$

где инфимум берется по всем $\rho$-допустимым $\pi$-представлениям $\varphi$ в точке $\zeta$. Если такого представления не сушествует, то полагаем $\rho_{I}(\zeta, \varphi)=\infty$. Подмодуль $I$ насыщен относительно әлемента $\varphi \in \mathbf{P}$, если для любой непрерьвной полунормы $\rho$ на $\mathbf{P}$ справедлива импликация:

$$
\Phi \text { - целая функция, } \quad|\Phi(\zeta)| \leqslant \rho_{I}(\zeta, \varphi) \quad \forall \zeta \in \mathbb{C} \quad \Rightarrow \quad \Phi=\text { const. }
$$

Подмодуль $I$ насыщен, если он насышен относительно любого элемента $\varphi \in \mathbf{P}$, $\varphi \in I(\widetilde{\lambda}) \forall \lambda \in \Lambda$.

Рассмотрим теперь $\mathbb{C}[l]$-подмодуль $J$ также ранга $k$. Представление вида $(7)$, где $u^{(1)}, \ldots, u^{(k)} \in J, c_{1}, \ldots, c_{k} \in O_{l}(\widetilde{\xi}), \widetilde{\xi}-l$-слой, содержащий $\zeta$, назьвается $l$-представлением. Аналогично тому, как это делалось выше, определяется понятие $\rho$-допустимого $l$-представления. Вводится функция

$$
\rho_{J}(\zeta, \varphi)=\inf \left\{1+\sum\left|c_{i}(\zeta)\right|\right\}
$$

где инфимум берется по всем $\rho$-допустимым $l$-представлениям $\varphi \in \mathbf{P}$ в точке $\zeta$. Подмодуль $J$ насыщен относительно әлемента $\varphi \in \mathbf{P}$, если для любой непрерывной полунормы $\rho$ на $\mathbf{P}$ справедлива импликация:

$$
\Phi \text { - целая функция, }|\Phi(\zeta)| \leqslant \rho_{J}(\zeta, \varphi) \forall \zeta \in \mathbb{C} \quad \Rightarrow \quad \Phi=\text { const. }
$$

Подмодуль $J$ насыщен, если он насыщен относительно любого элемента $\varphi \in \mathbf{P}$, $\varphi \in J(\widetilde{\xi}) \forall \xi \in \mathbb{C}$. 
ТЕОРема 2.2. Пусть $\mathbb{C}[\pi]-$ подмодуль $I$ и $\mathbb{C}[l]$-подмодуль $J$ связаньи соотношением $s J \subseteq I \subseteq J$. Тогда подмодули $I, J$ насъщены или нет одновременно.

Доказательство распадается на две части. В первой части устанавливается, что насышенность $I$ влечет насышенность $J$; во второй части проверяется обратное утверждение: насыщенность $J$ влечет насыщенность $I$.

ПРЕДЛОЖЕНИЕ 2.6. Насыщенность подмодуля I влечет насыщенность подмодуля $J$.

ЛЕмма 2.1. Пусть $\varphi \in \mathbf{P}, s-$ многочлен. Если $\mathbb{C}[\pi]-$ подмодуль $I$ насыщен относительно $s \varphi$, то он насыщен относительно $\varphi$.

ДоКАЗАТЕЛьство. Пусть подмодуль $I$ насыщен относительно $s \varphi, \rho$ - непрерывная полунорма на Р. Рассмотрим целую функцию $\Phi$, удовлетворяющую условию

$$
|\Phi(\zeta)| \leqslant \rho_{I}(\zeta, \varphi) \quad \forall \zeta \in \mathbb{C}
$$

Нужно показать, что $\Phi=$ const. Оценим $\rho_{I}(\zeta, \varphi)$, через $\rho_{I}(\zeta, s \varphi)$. По определению

$$
\rho_{I}(\zeta, s \varphi)=\inf \left\{1+\sum\left|c_{i}(\zeta)\right|\right\}
$$

где инфимум берется по всем $\rho$-допустимым $\pi$-представлениям $s \varphi$

$$
s \varphi=\sum_{i=1}^{k} c_{i} u^{(i)}
$$

в точке $\zeta$. Из (9) следует

$$
\varphi=\sum_{i=1}^{k} \frac{c_{i}}{s} u^{(i)}
$$

откуда

$$
\rho_{I}(\zeta, \varphi) \leqslant \inf \left\{1+\frac{1}{s(\zeta)} \sum\left|c_{i}(\zeta)\right|\right\},
$$

где инфимум берется по всем $\rho$-допустимым $\pi$-представлениям $(9)$. Пусть $D-$ замкнутый круг с центром в нуле, вне которого $s(\zeta) \geqslant 1$. При $\zeta \in \mathbb{C} \backslash D$ имеем

$$
\rho_{I}(\zeta, \varphi) \leqslant \inf \left\{1+\sum\left|c_{i}(\zeta)\right|\right\}=\rho_{I}(\zeta, s \varphi)
$$

В силу (8)

$$
|\Phi(\zeta)| \leqslant \rho_{I}(\zeta, s \varphi) \quad \forall \zeta \in \mathbb{C} \backslash D
$$

Далее, существует число $M \geqslant 1$, для которого $\max _{\zeta \in D}|\Phi(\zeta)| \leqslant M$. Учитывая, что $\rho_{I}(\zeta, s \varphi) \geqslant 1$, выводим $|\Phi(\zeta)| \leqslant M \rho_{I}(\zeta, s \varphi) \forall \zeta \in \mathbb{C}$ или $|\Phi(\zeta)| / M \leqslant \rho_{I}(\zeta, s \varphi)$ $\forall \zeta \in \mathbb{C}$. Поскольку подмодуль $I$ насьшен относительно $s \varphi$, заключаем, что $\Phi=$ const. Лемма доказана. 
ДоКАЗАТЕЛЬСТВО ПРЕДЛОЖЕНИЯ 2.6. Покажем, что для любого элемента $\varphi \in \mathbf{P}$ выполняется неравенство

$$
\rho_{J}(\zeta, \varphi) \leqslant \rho_{I}(\zeta, \varphi) \quad \forall \zeta \in \mathbb{C} \backslash \sigma,
$$

где $\sigma$-нулевое множество многочлена $s$. Пусть $\zeta \in \mathbb{C} \backslash \sigma$. Рассмотрим $\rho$-допустимое $\pi$-представление

$$
\varphi=\sum_{i=1}^{k} c_{i} u^{(i)} .
$$

Так как $\zeta \in \mathbb{C} \backslash \sigma$ и $I \subseteq J$, то это представление будет и $l$-представлением. Таким образом, множество $\rho$-допустимых $\pi$-представлений является частью множества $\rho$-допустимых $l$-представлений. Отсюда следует неравенство (10). Теперь предположим, что $I$ - насышенньй подмодуль. Пусть $\varphi \in \mathbf{P}, \varphi \in J(\widetilde{\xi}) \forall \xi \in \mathbb{C}$. Рассмотрим целую функцию $\Phi$, удовлетворяющую условию

$$
|\Phi(\zeta)| \leqslant \rho_{J}(\zeta, \varphi) \quad \forall \zeta \in \mathbb{C} .
$$

Нужно доказать, что $\Phi=$ const. В силу (10)

$$
|\Phi(\zeta)| \leqslant \rho_{I}(\zeta, \varphi) \quad \forall \zeta \in \mathbb{C} \backslash \sigma .
$$

Из определения $\rho_{I}(\zeta, \varphi)$ следует, что эта функция полунепрерывна сверху. С другой стороны, функция $|\Phi(\zeta)|$ непрерьвна. Поэтому неравенство (11) распространяется с $\mathbb{C} \backslash \sigma$ на все $\mathbb{C}$. Итак

$$
|\Phi(\zeta)| \leqslant \rho_{I}(\zeta, \varphi) \quad \forall \zeta \in \mathbb{C} .
$$

По предположению выполняется соотношение $s J \subseteq I$. Из этого соотношения вытекает, что условие $\varphi \in J(\widetilde{\xi}) \forall \xi \in \mathbb{C}$ влечет $s \varphi \in I(\widetilde{\widetilde{\lambda}}) \forall \lambda \in \Lambda$. Так как подмодуль $I$ насышен, то он будет насышен относительно $s \varphi$. По лемме 2.1 подмодуль $I$ насышен и относительно $\varphi$. На основании неравенства (12) заключаем, что $\Phi=$ const. Это справедливо для любой непрерывной полунормы $\rho$ на $\mathbf{P}$ и любого элемента $\varphi \in \mathbf{P}$, $\varphi \in I(\widetilde{\xi}) \forall \xi \in \mathbb{C}$. Следовательно, $J$ - насышенный подмодуль. Предложение доказано.

ПРЕДЛОЖЕНИЕ 2.7. Насыщенность подмодуля $Ј$ влечет насыщенность подмодуля $I$.

Лемма 2.2. Пусть $s-$ многочлен. Для любой непрерывной полунормы $\rho$ на $\mathbf{P}$ существует другая непрерывная полунорма $\rho^{\prime}$ такая, что справедлива импликация

$$
\rho^{\prime}(f) \leqslant 1 \quad \Rightarrow \quad \rho(s f) \leqslant 1 .
$$

ДоКАЗАТЕЛЬСтво. Пусть

$$
U=\{f: \rho(f) \leqslant 1\},
$$

$U$ - абсолютно выпуклая замкнутая окрестность нуля в $\mathbf{P}$. Так как операция умножения на многочлен непрерывна в $\mathbf{P}$, то существует абсолютно выпуклая замкнутая окрестность нуля $U^{\prime}$ такая, что $s U^{\prime} \subseteq U$. Пусть $\rho^{\prime}(f)=\inf \left\{\lambda>0: f \in \lambda U^{\prime}\right\}$, $\rho^{\prime}$ - непрерывная полунорма на Р. Покажем, что выполняется импликация (13). Действительно, если $\rho^{\prime}(f) \leqslant 1$, то $f \in U^{\prime}$. Тогда $s f \in U$, что равносильно $\rho(s f) \leqslant 1$. Лемма доказана. 
Лемма 2.3. Пусть $D$ - замкнутый круг с иентром в нуле, вне которого $|s(\zeta)| \geqslant 1$. Для любой непрерывной полунормы $\rho$ на $\mathbf{P}$ существует другая непрерывная полунорма $\rho^{\prime}$ такая, что

$$
\rho_{I}(\zeta, \varphi) \leqslant \rho_{J}^{\prime}(\zeta, \varphi) \quad \forall \zeta \in \mathbb{C} \backslash D
$$

ДокАЗАТЕЛьство. По лемме 2.2 подбираем непрерывную полунорму $\rho^{\prime}$, для которой выполняется импликация (13). Пусть $\zeta \in \mathbb{C} \backslash D$. Рассмотрим $\rho^{\prime}$-допустимое $l$-представление

$$
\varphi=\sum_{i=1}^{k} c_{i} u^{(i)}
$$

в точке $\zeta$. Запишем это представление в виде

$$
\varphi=\sum_{i=1}^{k} \frac{c_{i}}{s} v^{(i)}
$$

где $v^{(1)}=s u^{(1)}, \ldots, v^{(k)}=s u^{(k)}$. В силу включения $s J \subseteq I$

$$
v^{(1)}, \ldots, v^{(k)} \in I .
$$

Кроме того, при $\zeta \notin \sigma$ коэффициенты $c_{i} / s \in O_{\pi}(\widetilde{\lambda})$, где $\widetilde{\lambda}-\pi$-слой, содержащий $\zeta$. Таким образом, (15) есть $\pi$-представление $\varphi$ в точке $\zeta$. В силу импликации (13) это представление является $\rho$-допустимым. Множество всех $\rho$-допустимых $\pi$-представлений шире, чем множество представлений вида (15), поэтому

$$
\rho_{I}(\zeta, \varphi) \leqslant \inf \left\{1+\frac{1}{s(\zeta)} \sum\left|c_{i}(\zeta)\right|\right\}
$$

где инфимум берется по всем $l$-представлениям вида (14). Далее, при $\zeta \in \mathbb{C} \backslash D$ $|s(\zeta)| \geqslant 1$, поэтому при $\zeta \in \mathbb{C} \backslash D$ имеем

$$
\rho_{I}(\zeta, \varphi) \leqslant \inf \left\{1+\frac{1}{s(\zeta)} \sum\left|c_{i}(\zeta)\right|\right\} \leqslant \inf \left\{1+\sum\left|c_{i}(\zeta)\right|\right\}=\rho_{J}^{\prime}(\zeta, \varphi)
$$

Итак, $\rho_{I}(\zeta, \varphi) \leqslant \rho_{J}^{\prime}(\zeta, \varphi) \forall \zeta \in \mathbb{C} \backslash D$. Лемма доказана.

ДОКАЗАТЕЛЬСТВО ПРЕДЛОЖЕНИЯ 2.7. Пусть Подмодуль $J$ насьщен и $\varphi \in \mathbf{P}$, $\varphi \in I(\widetilde{\lambda}) \forall \lambda \in \Lambda$. Рассмотрим целую функцию $\Phi$, удовлетворяюшую условию

$$
|\Phi(\zeta)| \leqslant \rho_{I}(\zeta, \varphi) \quad \forall \zeta \in \mathbb{C} .
$$

Нужно показать, что $\Phi=$ const. По лемме 2.3 существует непрерывная полунорма $\rho^{\prime}$ такая, что

$$
\rho_{I}(\zeta, \varphi) \leqslant \rho_{J}^{\prime}(\zeta, \varphi) \quad \forall \zeta \in \mathbb{C} \backslash D
$$

поэтому $|\Phi(\zeta)| \leqslant \rho_{J}^{\prime}(\zeta, \varphi) \forall \zeta \in \mathbb{C} \backslash D$. Пусть $M \geqslant \max _{D}|\Phi|, M \geqslant 1$. Так как $\rho_{J}^{\prime}(\zeta, \varphi) \geqslant 1$ и $M \geqslant 1$, то $|\Phi(\zeta)| \leqslant M \rho_{J}^{\prime}(\zeta, \varphi) \forall \zeta \in \mathbb{C}$ или

$$
\frac{|\Phi(\zeta)|}{M} \leqslant \rho_{J}^{\prime}(\zeta, \varphi) \quad \forall \zeta \in \mathbb{C} .
$$

По условию $\varphi \in \mathbf{P}, \varphi \in I(\widetilde{\lambda}) \forall \lambda \in \Lambda$. Учитывая включение $I \subseteq J$, получаем, что $\varphi \in J(\widetilde{\xi}) \forall \xi \in \mathbb{C}$. Насыщенность подмодуля $J$ влечет его насышенность относительно $\varphi$. Это значит, что неравенство (16) влечет $\Phi=$ const. Насьщенность $I$ установлена. 
2.3. Критерий обильности. Как и в случае $q=1$, проверка обильности замкнутого $\mathbb{C}[\pi]$-подмодуля в $\mathbf{P}$ сводится к проверке его устойчивости и насьшенности.

КРИТЕРИЙ ОБИЛЬНОСТИ. Для того чтобы замкнутый $\mathbb{C}[\pi]-$ подмодуль I был обильныцм, необходимо и достаточно, чтобы он был устойчив и насыщен.

ДокАЗАТЕльСтво. Пусть $I$ - произвольный замкнутый $\mathbb{C}[\pi]$-подмодуль в $\mathbf{P}$, $J$ - замкнутый $\mathbb{C}[l]$-подмодуль в $\mathbf{P}$, порождаемый подмодулем $I$. По теореме редукции подмодули $I, J$ обильны или нет одновременно. По критерию обильности замкнутых $\mathbb{C}[l]$-подмодулей в $\mathbf{P}[11]$ обильность $\mathbb{C}[l]$-подмодуля $J$ равносильна его устойчивости и насыщенности. Из теорем 2.1 и 2.2 , в свою очередь, вытекает, что обильность $\mathbb{C}[\pi]$-подмодуля $I$ равносильна его устойчивости и насыщенности. Утверждение доказано.

\section{§3. $\pi$-свертка}

3.1. Дуальные отображения. Пусть $\Omega$ - область Рунге в $\mathbb{C}^{q} ; H(\Omega)$ - пространство функций, голоморфных в $\Omega$, с топологией компактной сходимости; $H^{*}(\Omega)$ - пространство, сильное сопряженное к $H(\Omega) ; T$ - преобразование Лапласа функционалов из $H^{*}(\Omega)$, которое каждому функционалу $S \in H^{*}(\Omega)$ ставит в соответствие целую функцию экспоненциального типа $\varphi(\lambda)=\left\langle S, e^{\langle\lambda, h\rangle}\right\rangle ; P(\Omega)-$ полный образ $T$. Известно, что отображение $T$ является взаимно однозначньм $[16 ; \S 12$, лемма 12.1]. Считаем, что $P(\Omega)$ наделено локально выпуклой топологией, индуцированной отображением $T$ из $H^{*}(\Omega)$.

Если $\Omega$ - выпуклая область в $\mathbb{C}^{q}$, то пространство $P(\Omega)$ относится к пространствам типа $\left(L N^{*}\right)$. Его топология может быть описана как топология индуктивного предела банаховых пространств

$$
P\left(d_{k}\right)=\left\{\varphi \in O\left(\mathbb{C}^{q}\right):\|\varphi\|_{k}<\infty\right\}
$$

с нормами

$$
\|\varphi\|_{k}=\sup _{\lambda \in \mathbb{C}^{q}} \frac{|\varphi(\lambda)|}{\exp H_{d_{k}}(\lambda)}
$$

где $d_{1} \Subset d_{2} \Subset \cdots-$ последовательность компактов, исчерпываюшая $\Omega, H_{d_{k}}(\lambda)=$ $\sup _{\omega \in d_{k}} \operatorname{Re}\langle\lambda, \omega\rangle$ - опорная функция компакта $d_{k}$ в смысле комплексного анализа $[16 ; \S 12$, п. 7$]$.

В случае одной переменной будем использовать другое описание этой топологии. Пусть $G$ - выпуклая область в $\mathbb{C} ; H_{G}(\zeta)$ - опорная функция области $G$ в смысле комплексного анализа; $\mathfrak{M}_{G}-$ класс положительных на $\mathbb{C}$ функций $m(\zeta)$ со свойствами:

1) $m(\zeta) \exp \left\{-H_{d}(\zeta)\right\} \rightarrow \infty$ при $\zeta \rightarrow \infty$ для любого компакта $d \subset G$;

$2) \inf _{\zeta \in d} m(\zeta)>0$ для любого компакта $d \subset \mathbb{C}$.

Совокупность множеств $V_{m}=\{\varphi \in P(G):|\varphi(\zeta)| \leqslant m(\zeta)\}, m \in \mathfrak{M}_{G}$, образует фундаментальную систему окрестностей нуля в пространстве $P(G)[4 ; \S 3$, п. 3$]$.

Пространство $H(\Omega)$ является рефлексивным, значит, существует линейный топологический изоморфизм $\omega: H(\Omega) \rightarrow P^{*}(\Omega)[17 ; \S 1]$. При этом если $f^{\odot}=\omega(f)$ и $\varphi=T(S)$, то

$$
\left\langle f^{\odot}, \varphi\right\rangle=\langle S, f\rangle
$$


(см. [17; предложение 1.1]).

Пусть $u: H(\Omega) \rightarrow H(G)$ - произвольное линейное непрерывное отображение. Сопряженное отображение $u^{*}: H^{*}(G) \rightarrow H^{*}(\Omega)$ функционалу $S \in H^{*}(G)$ ставит в соответствие функционал из $H^{*}(\Omega)$, действующий по правилу $f \rightarrow\langle S, u(f)\rangle$. Преобразование Лапласа $T$ индуцирует операцию $u^{\odot}: P(G) \rightarrow P(\Omega)$, которая функцию $\varphi \in P(G), \varphi(\lambda)=\left\langle S, e^{\langle\lambda, h\rangle}\right\rangle$, переводит в функцию $\psi \in P(\Omega), \psi(\zeta)=$ $\left\langle S, u\left(e^{\zeta z}\right)\right\rangle$. Отображение $u^{\odot}$ назьвают дуальным по отношению к прямому отображению $u[17 ; \S 1$, п. 1.3]. Прямая и дуальная операции связаны соотношением:

$$
\left\langle f^{\odot}, u^{\odot}(\varphi)\right\rangle=\langle S, u(f)\rangle
$$

где $f \in H(\Omega), S \in H^{*}(G), f^{\odot}=\omega(f), \varphi=T(S)$ [17; предложение 1.2]. Стандартным образом [17], [18] доказьвается следующее утверждение.

$1^{\circ}$. Пусть $\left\{u_{k}\right\}$ - семейство линейных непрерывных отобрахсений из $H(\Omega)$ в $H(G)$. Для того чтоби семейство $\left\{u_{k}\right\}$ било равностепенно непрерьвным, необходимо и достаточно, чтобы равностепенно непрерывным было семейство дуальных отображений $\left\{u_{k}^{\odot}\right\}$.

Следующее утверждение для дуальных операций проистекает от соответствующего утверждения для сопряженных операций.

$2^{\circ}$. Если $v$ - линейный непрерывный оператор, действующий в $H(\Omega), v^{\odot}-$ дуальный по отношению $к v$ оператор, действующий в $P(\Omega)$, то

$$
(u \circ v)^{\odot}=v^{\odot} \circ u^{\odot} .
$$

3.2. $\pi$-симметризация. Понятие $\pi$-симметризации для одного многочлена $\pi$ введено И.. Красичковьп-Терновским в [19]. Ниже определяется аналог этого понятия для случая системы многочленов $\pi_{1}, \ldots, \pi_{q}$.

Пусть $\Phi=\Phi(\lambda)$ - целая функция $q$ переменных $\lambda_{1}, \ldots, \lambda_{q}$. Выберем $\zeta \in \mathbb{C}$ и обозначим через $\langle\zeta\rangle$ декартово произведение $\langle\zeta\rangle_{1} \times \cdots \times\langle\zeta\rangle_{q}$, упорядоченное произвольным образом, где $\langle\zeta\rangle_{p}-$ произвольное упорядочение $\pi_{p}$-слоя, содержащего $\zeta$, причем каждая точка слоя присутствует в $\langle\zeta\rangle_{p}$ столько раз, какова ее кратность. Рассмотрим функцию

$$
F(\zeta)=\frac{1}{m} \sum_{\lambda \in\langle\zeta\rangle} \Phi(\lambda)
$$

где $m=m_{1} \times \cdots \times m_{q}, m_{p}$-степень многочлена $\pi_{p}$. Из определения множества $\langle\zeta\rangle$ вытекает, что эту функцию можно представить в виде

$$
F(\zeta)=\frac{1}{m} \sum_{\lambda_{1} \in\langle\zeta\rangle_{1}} \ldots \sum_{\lambda_{q} \in\langle\zeta\rangle_{q}} \Phi\left(\lambda_{1}, \ldots, \lambda_{q}\right) .
$$

Функция

$$
\frac{1}{m_{p}} \sum_{\lambda_{p} \in\langle\zeta\rangle_{p}} \Phi\left(\lambda_{1}, \ldots, \lambda_{p}, \ldots, \lambda_{q}\right)
$$

является целой функцией переменных $\lambda_{1}, \ldots, \pi_{p}(\zeta), \ldots, \lambda_{q}[14 ;$ предложение 2.2$]$. Из представления (19) вытекает, что функция $F(\zeta)$ является целой функцией переменных $\pi_{1}(\zeta), \ldots, \pi_{q}(\zeta)$. Последнее означает, что функция $F(\zeta) \pi$-симметрична 
на $\mathbb{C}$. Следуя И.. Красичкову-Терновскому [14], функцию $F(\zeta)$ будем называть $\pi$-симметризацией функции $\Phi$ и обозначать $(\operatorname{sym} \Phi(\lambda))(\zeta)$, или $\left(\operatorname{sym}_{\lambda} \Phi(\lambda)\right)(\zeta)$, если необходимо указать переменный вектор, по которому осуществляется симметризация.

Если $\Phi(\lambda)=\Phi_{1}\left(\lambda_{1}\right) \times \cdots \times \Phi_{q}\left(\lambda_{q}\right)$, где $\Phi_{p}$ - целая функция одной переменной, то из представления (19) вытекает, что $\operatorname{sym} \Phi=\operatorname{sym} \Phi_{1} \times \cdots \times \operatorname{sym} \Phi_{q} \cdot$ Здесь $\operatorname{sym} \Phi_{p}-$ $\pi_{p}$-симметризация функции $\Phi_{p}$. В частности, $\pi$-симметризация $\pi_{\gamma}$ одночлена $\lambda^{\gamma}=$ $\lambda_{1}^{\gamma_{1}} \times \cdots \times \lambda_{q}^{\gamma_{q}}$ совпадает с произведением $\pi_{\gamma_{1}} \times \cdots \times \pi_{\gamma_{q}}$, где $\pi_{\gamma_{p}}-\pi_{p}$-симметризация одночлена $\lambda_{p}^{\gamma_{p}}$. Заметим, что для всех $\lambda_{p} \in\langle\zeta\rangle_{p}$ имеет место оценка

$$
\left|\lambda_{p}\right| \leqslant|\zeta|\left(1+\varepsilon_{p}(\zeta)\right)+\text { const }
$$

где $\varepsilon_{p}(\zeta)$ - неотрицательные ограниченные на $\mathbb{C}$ функции, $\varepsilon_{p}(\zeta) \rightarrow 0$ при $\zeta \rightarrow \infty$. Следовательно, при $\zeta \neq 0$ имеем

$$
\left|\pi_{\gamma_{p}}(\zeta)\right| \leqslant \frac{1}{m_{p}} \sum_{\lambda_{p} \in\langle\zeta\rangle_{p}}\left|\lambda_{p}^{\gamma_{p}}\right| \leqslant|\zeta|^{\gamma_{p}}\left(1+\varepsilon_{p}(\zeta)+\frac{\text { const }}{|\zeta|}\right)^{\gamma_{p}}
$$

Из этой оценки вытекает, что $\pi_{\gamma_{p}}$ - многочлен, степень которого не превосходит числа $\gamma_{p}$. Значит, степень многочлена $\pi_{\gamma}$ не превосходит числа $|\gamma|=\gamma_{1}+\cdots+\gamma_{q}$.

Пусть $O$ - открытая круговая окрестность нуля в $\mathbb{C} ; \mathbf{O}-$ открытая поликруговая окрестность нуля в $\mathbb{C}^{q}$, столь “малая", что

$$
\left\{\zeta: \zeta=\lambda_{1}+\cdots+\lambda_{q}, \lambda=\left(\lambda_{1}, \ldots, \lambda_{q}\right) \in \mathbf{O}\right\} \subseteq O .
$$

При этом полирадиус $(\sigma, \ldots, \sigma)$ поликруга О и радиус $\rho$ круга $O$ связаны неравенством $\sigma q \leqslant \rho$.

При доказательстве следующего предложения символом $P(U)$, где $U$ - замкнутая поликруговая окрестность нуля в $\mathbb{C}^{q}$ (соответственно $P(V)$, где $V$ - замкнутая круговая окрестность нуля в $\mathbb{C}$ ), обозначаем банахово пространство

$$
\left\{\Phi \in O\left(\mathbb{C}^{q}\right):\|\Phi\|<\infty\right\} \quad(\text { соответственно } \quad\{F \in O(\mathbb{C}):\|F\|<\infty\})
$$

с нормой

$$
\|\Phi\|=\sup _{\lambda \in \mathbb{C}^{q}} \frac{|\Phi(\lambda)|}{\exp H_{U}(\lambda)} \quad\left(\text { соответственно } \quad\|F\|=\sup _{\zeta \in \mathbb{C}} \frac{|F(\zeta)|}{\exp H_{V}(\zeta)}\right) .
$$

ПРЕДЛОЖЕНИЕ 3.1. Оператор $\pi$-симметризаиии $\mathrm{sym}: P(\mathbf{O}) \rightarrow P(O)$ является линейным и непрерывным.

ДокАЗАТЕЛьство. Линейность оператора $\pi$-симметризации очевидна. Сначала докажем его секвенциальную непрерьвность. Пусть $\Phi_{k}(\lambda) \rightarrow 0$ в $P(\mathbf{O})$. Нам достаточно показать, что $F_{k}(\zeta)=\left(\operatorname{sym} \Phi_{k}(\lambda)\right)(\zeta) \rightarrow 0$ в $P(O)$. По свойствам пространств типа $\left(L N^{*}\right)$ [16; теорема 2.2, следствие 2.3] найдется $\sigma_{1}<\sigma$ такое, что $\Phi_{k}(\lambda) \rightarrow 0$ по норме пространства $P(U)$, где $U \subset \mathbf{O}$ - замкнутая поликруговая окрестность нуля полирадиуса $\left(\sigma_{1}, \ldots, \sigma_{1}\right)$. Значит, справедливы оценки:

$$
\left|\Phi_{k}(\lambda)\right| \leqslant\left\|\Phi_{k}\right\| \exp H_{U}(\lambda)=\left\|\Phi_{k}\right\| \exp \left(\sigma_{1} \sum_{p}\left|\lambda_{p}\right|\right)
$$


где $\left\|\Phi_{k}\right\| \rightarrow 0$. Отсюда получаем

$$
\left|F_{k}(\zeta)\right| \leqslant \frac{1}{m} \sum_{\lambda \in\langle\zeta\rangle}\left|\Phi_{k}(\lambda)\right| \leqslant \frac{1}{m} \sum_{\lambda \in\langle\zeta\rangle}\left\|\Phi_{k}\right\| \exp \left(\sigma_{1} \sum_{p}\left|\lambda_{p}\right|\right) .
$$

Из оценки (20) вытекает, что

$$
\begin{aligned}
\left|F_{k}(\zeta)\right| & \leqslant\left\|\Phi_{k}\right\| \exp \left(\sigma_{1} \sum_{p}|\zeta|\left(1+\varepsilon_{p}(\zeta)\right)+\text { const }\right) \\
& \leqslant\left\|\Phi_{k}\right\| \exp \left(\sigma_{1} q|\zeta|(1+\varepsilon(\zeta))+\text { const }\right) \leqslant M\left\|\Phi_{k}\right\| \exp \rho_{1}|\zeta|
\end{aligned}
$$

где $\varepsilon(\zeta)=\max _{p} \varepsilon_{p}(\zeta), M>0, \sigma_{1}<\rho_{1}<\rho$. Следовательно, $F_{k} \rightarrow 0$ по норме пространства $P(V)$. Здесь $V$ - замкнутая круговая окрестность нуля в $\mathbb{C}$ радиуса $\rho_{1}$. Так как отображение вложения $P(V) \rightarrow P(O)$ непрерьвно, то $F_{k} \rightarrow 0$ в топологии $P(O)$. Секвенциальная непрерьвность оператора $\pi$-симметризации sym: $P(\mathbf{O}) \rightarrow P(O)$ доказана. Осталось напомнить, что пространство $P(\mathbf{O})$ является пространством типа $\left(L N^{*}\right)$. Следовательно, секвенциальная непрерывность этого оператора влечет его непрерывность [20], [16; теорема 3.3]. Предложение доказано.

3.3. $\pi$-сдвиг. Пусть $G, \Omega$ - односвязные области в $\mathbb{C} ; O$ - открытая круговая окрестность нуля в $\mathbb{C}$ радиуса $\rho>0$. При этом $G+O \subset \Omega$. Обозначим через $\mathbf{O}$ открытую поликруговую окрестность нуля в $\mathbb{C}^{q}$ полирадиуса $(\sigma, \ldots, \sigma), \sigma>0$, удовлетворяющую условию (21).

Рассмотрим последовательность $\left\{F_{k}(\zeta, h)\right\}_{k=0}^{\infty}$ целых функций одной переменной $\zeta$, зависящих от параметра $h$, принимающего значения из компакта $d \subset \mathbf{O}$. Следуюшая лемма навеяна контактами с И. $\Phi$. Красичковым-Терновским.

ЛЕмма 3.1. Пусть $F_{k}(\zeta, h) \rightarrow F_{0}(\zeta, h)$ в $P(O)$ равномерно по $h$ из компакma $d \subset$ O. Тогда для любого компакта $d^{\prime} \subset G$

$$
e^{\zeta z} F_{k}(\zeta, h) \rightarrow e^{\zeta z} F_{0}(\zeta, h)
$$

в $P(\Omega)$ равномерно по $h \in d u z \in d^{\prime}$.

ДокАЗАТЕльство. Выберем произвольньй компакт $d^{\prime} \subset G$ и круговую окрестность $о$ нуля радиуса $\varepsilon>0$ столь малую, что $d^{\prime}+o \subseteq G$. Совокупность множеств $V_{m}=\{\varphi \in P(o+O):|\varphi(\zeta)| \leqslant m(\zeta)\}, m \in \mathfrak{M}_{o+O}$, образует фундаментальную систему окрестностей нуля в пространстве $P(o+O)$ (см. п. 3.1). Выберем произвольную окрестность $V_{m}, m \in \mathfrak{M}_{o+O}$. Положительная функция $\widehat{m}(\zeta)=m(\zeta) \exp (-\varepsilon|\zeta|)$ принадлежит классу $\mathfrak{M}_{O}$. Значит, множество $V_{\widehat{m}}=$ $\{\varphi \in P(O):|\varphi(\zeta)| \leqslant m(\zeta) \exp (-\varepsilon|\zeta|)\}$ является окрестностью нуля в пространстве $P(O)$. Так как $F_{k}(\zeta, h) \rightarrow F_{0}(\zeta, h)$ в $P(O)$ равномерно по $h \in d$, то для всех $k \geqslant k_{0}$ и $h \in d$ имеем $\left|F_{k}(\zeta, h)-F_{0}(\zeta, h)\right| \leqslant m(\zeta) \exp (-\varepsilon|\zeta|)$. Отсюда вытекает, что для всех $k \geqslant k_{0}, h \in d$ и $z \in$ о выполнено $\left|e^{\zeta z}\left(F_{k}(\zeta, h)-F_{0}(\zeta, h)\right)\right| \leqslant m(\zeta)$, т.е. $e^{\zeta z}\left(F_{k}(\zeta, h)-F_{0}(\zeta, h)\right) \in V_{m}$. Следовательно, $e^{\zeta z} F_{k}(\zeta, h) \rightarrow e^{\zeta z} F_{0}(\zeta, h)$ в топологии пространства $P(o+O)$. Причем сходимость равномерная по $h \in d$ и $z \in o$. Подберем точки $a_{1}, \ldots, a_{n} \in d^{\prime}$ так, чтобы множества $a_{1}+o, \ldots, a_{n}+o$ покрьвали компакт $d^{\prime}$. Отображение $P(o+O) \rightarrow P\left(a_{i}+o+O\right), F \rightarrow e^{a_{i} \zeta} F$, является линейным топологическим изоморфизмом. Следовательно, $e^{\zeta\left(a_{i}+z\right)} F_{k}(\zeta, h) \rightarrow e^{\zeta\left(a_{i}+z\right)} F_{0}(\zeta, h)$ 
в топологии $P\left(a_{i}+o+O\right)$, причем сходимость равномерная по $h \in d$ и $z \in o$. Отсюда вытекает, что $e^{\zeta z} F_{k}(\zeta, h) \rightarrow e^{\zeta z} F_{0}(\zeta, h)$ в топологии $P\left(a_{i}+o+O\right)$, причем сходимость равномерная по $h \in d$ и $z \in a_{i}+o$. Вложения $P\left(a_{i}+o+O\right) \subseteq P(\Omega)$ являются непрерьвными, значит, $e^{\zeta z} F_{k}(\zeta, h) \rightarrow e^{\zeta z} F_{0}(\zeta, h)$ в топологии $P(\Omega)$, причем сходимость равномерная по $h \in d$ и $z \in d^{\prime}$. Лемма доказана.

ПРЕДЛОЖЕНИЕ 3.2. Пусть $\omega: H(\Omega) \rightarrow P^{*}(\Omega)$ - линейный топологический изоморфизм, $f \in H(\Omega), f^{\odot}=\omega(f)$ (см. п. 3.1). Ряд

$$
\sum_{|\gamma|=0}^{\infty} \frac{\left(\pi_{\gamma}(D) f\right)(z)}{\gamma !} h^{\gamma}
$$

сходится $\kappa f(z, h):=\left\langle f^{\odot}, e^{\zeta z}\left(\operatorname{sym}_{\lambda} e^{\langle\lambda, h\rangle}\right)(\zeta)\right\rangle$ равномерно на компактах из $G \times \mathbf{O}, u$, следовательно, его сумма является аналитической функиией на $G \times \mathbf{O}$.

ДокАЗАТЕЛьство. Пусть $\pi_{\gamma}^{*}(D)$ - оператор $H^{*}(\Omega) \rightarrow H^{*}(\Omega)$, сопряженньй к дифференциальному оператору $\pi_{\gamma}(D) ; \delta_{z}-$ функционал из $H^{*}(\Omega)$, действующий по правилу $f \rightarrow f(z), z \in \Omega$. Напомним, что преобразование Лапласа $T: H^{*}(\Omega) \rightarrow P(\Omega)$ функционалу $S \in H^{*}(\Omega)$ ставит в соответствие целую функцию $\varphi(\zeta)=\left\langle S, e^{\zeta z}\right\rangle$. Из равенств

$$
\left\langle\frac{\pi_{\gamma}^{*}(D) \delta_{z_{0}}}{\gamma !}, e^{\zeta z}\right\rangle=\frac{1}{\gamma !}\left\langle\delta_{z_{0}}, \pi_{\gamma}(D) e^{\zeta z}\right\rangle=\frac{\pi_{\gamma}(\zeta)}{\gamma !} e^{\zeta z_{0}}
$$

вытекает, что

$$
T\left(\frac{\pi_{\gamma}^{*}(D) \delta_{z_{0}}}{\gamma !}\right)=\frac{\pi_{\gamma}(\zeta)}{\gamma !} e^{\zeta z_{0}}
$$

Используя это соотношение и соотношение (17), для любого $z \in \Omega$ имеем

$$
\begin{aligned}
f^{(k)}(z, h) & :=\sum_{|\gamma|=0}^{k} \frac{\left(\pi_{\gamma}(D) f\right)(z)}{\gamma !} h^{\gamma}=\left\langle\delta_{z}, \sum_{|\gamma|=0}^{k} \frac{\left(\pi_{\gamma}(D) f\right)}{\gamma !} h^{\gamma}\right\rangle \\
& =\left\langle\sum_{|\gamma|=0}^{k} \frac{\pi_{\gamma}^{*}(D) \delta_{z}}{\gamma !} h^{\gamma}, f\right\rangle=\left\langle f^{\odot}, \sum_{|\gamma|=0}^{k} \frac{\pi_{\gamma}(\zeta) h^{\gamma}}{\gamma !} e^{\zeta z}\right\rangle
\end{aligned}
$$

Пусть $d-$ компакт в О. Частичные суммы

$$
\Phi_{k}(\lambda, h)=\sum_{|\gamma|=0}^{k} \frac{\lambda^{\gamma} h^{\gamma}}{\gamma !}
$$

сходятся к $e^{\langle\lambda, h\rangle}$ в топологии пространства $H\left(\mathbb{C}^{q}\right)$ равномерно по $h \in d$. Здесь $\langle\lambda, h\rangle=\sum_{p=1}^{q} \lambda_{p} h_{p}$. При этом для любого $h \in d$ выполняются неравенства

$$
\left|\Phi_{k}(\lambda, h)-e^{\langle\lambda, h\rangle}\right| \leqslant \exp \left(\sum_{p=1}^{q}\left|\lambda_{p} h_{p}\right|\right) \leqslant \exp \left(\sigma_{d} \sum_{p=1}^{q}\left|\lambda_{p}\right|\right),
$$


где $\sigma_{d}=\max _{h \in d} \max _{p=1, \ldots, q}\left|h_{p}\right|<\sigma$. Следовательно, равномерно по $h \in d$

$$
\sup _{\lambda \in \mathbb{C}^{q}} \frac{\left|\Phi_{k}(\lambda, h)-e^{\langle\lambda, h\rangle}\right|}{\exp H_{U}(\lambda)} \rightarrow 0, \quad k \rightarrow \infty .
$$

Здесь $U$ - замкнутая поликруговая окрестность нуля полирадиуса $\left(\sigma^{\prime}, \ldots, \sigma^{\prime}\right)$, $\sigma_{d}<\sigma^{\prime}<\sigma ; H_{U}(\lambda)=\sigma^{\prime} \sum_{p=1}^{q}\left|\lambda_{p}\right|$ - опорная функция $U$ в смысле комплексного анализа. Таким образом, частичные суммы $\Phi_{k}(\lambda, h)$ сходятся к $e^{\langle\lambda, h\rangle}$ по норме пространства $P(U)$ равномерно по $h \in d$. Значит, $\Phi_{k}(\lambda, h) \rightarrow e^{\langle\lambda, h\rangle}$ в топологии $P(\mathbf{O})$ равномерно по $h \in d$. По предложению 3.1

$$
\left.\operatorname{sym}_{\lambda} \Phi_{\mathrm{k}}(\lambda, h)\right)(\zeta)=\sum_{|\gamma|=0}^{k} \frac{\pi_{\gamma}(\zeta)}{\gamma !} h^{\gamma} \rightarrow\left(\operatorname{sym}_{\lambda} e^{\langle\lambda, h\rangle}\right)(\zeta)
$$

в топологии $P(O)$ равномерно по $h \in d$. Пусть $d^{\prime}$ - компакт в $G$. В силу леммы 3.1 $e^{\zeta z}\left(\operatorname{sym}_{\lambda} \Phi_{k}(\lambda, h)\right)(\zeta) \rightarrow e^{\zeta z}\left(\operatorname{sym}_{\lambda} e^{\langle\lambda, h\rangle}\right)(\zeta)$ в топологии пространства $P(\Omega)$. При этом сходимость равномерна по $(z, h) \in d^{\prime} \times d$. Непрерывность функционала $f^{\odot}$ влечет

$$
f^{(k)}(z, h)=\sum_{|\gamma|=0}^{k} \frac{\left(\pi_{\gamma}(D) f\right)(z)}{\gamma !} h^{\gamma} \rightarrow\left\langle f^{\odot}, e^{\zeta z}\left(\operatorname{sym}_{\lambda} e^{\langle\lambda, h\rangle}\right)(\zeta)\right\rangle
$$

сходимость равномерна по $(z, h) \in d^{\prime} \times d$. Это верно для любых компактов $d^{\prime} \subset G$, $d \subset$ О. Поэтому функция $\left\langle f^{\odot}, e^{\zeta z}\left(\operatorname{sym}_{\lambda} e^{\langle\lambda, h\rangle}\right)(\zeta)\right\rangle$ голоморфна на декартовом произведении $G \times$ О. Предложение доказано.

Функция $f(z, h)$ назьвается $\pi$-сдвигом $f$ на шаг $h$, а отображение $T_{h}: H(\Omega) \rightarrow$ $H(G)$, которое функции $f \in H(\Omega)$ ставит в соответствие функцию $f(z, h) \in H(G)$ ( $h$ фиксировано в О), называется оператором $\pi$-сдвига на шаг $h$. Оператор $\pi$-сдвига в ситуации $q=1$ введен И. $\Phi$. Красичковым-Терновским в работе [19].

Наиболее простой вид $\pi$-сдвиг функции $f \in H(\Omega)$ на шаг $h$ приобретает в условиях, когда $\pi_{1}(\zeta)=\zeta^{m_{1}}, \ldots, \pi_{q}(\zeta)=\zeta^{m_{q}}$, где $\mu=\left(m_{1}, \ldots, m_{q}\right)$ - фиксированный вектор из $\mathbb{Z}_{+}^{q}$ с ненулевыми компонентами. В этом случае для любого $\zeta \in \mathbb{C}$ имеем

$$
\begin{gathered}
\langle\zeta\rangle_{1}=\left\{\zeta, \varepsilon_{1} \zeta, \ldots, \varepsilon_{1}^{m_{1}-1} \zeta\right\}, \ldots,\langle\zeta\rangle_{q}=\left\{\zeta, \varepsilon_{q} \zeta, \ldots, \varepsilon_{q}^{m_{q}-1} \zeta\right\}, \\
\text { где } \varepsilon_{p}=\exp \frac{2 \pi}{m_{p}} i . \text { Следовательно, для произвольного } \gamma \in \mathbb{Z}_{+}^{q} \text { получим } \\
\pi_{\gamma}(\zeta)=\frac{1}{m} \sum_{\lambda \in\langle\zeta\rangle} \lambda^{\gamma}=\frac{1}{m} \zeta^{|\gamma|} \sum_{\alpha<\mu} \varepsilon^{\alpha \gamma},
\end{gathered}
$$

где $m=\prod_{p} m_{p}, \lambda^{\gamma}=\prod_{p} \lambda_{p}^{\gamma_{p}},|\gamma|=\gamma_{1}+\cdots+\gamma_{q}$. При этом

$$
\sum_{\alpha<\mu} \varepsilon^{\alpha \gamma}=\sum_{\alpha_{1}=0}^{m_{1}-1} \cdots \sum_{\alpha_{q}=0}^{m_{q}-1} \varepsilon_{1}^{\alpha_{1} \gamma_{1}} \times \cdots \times \varepsilon_{q}^{\alpha_{q} \gamma_{q}}=\prod_{p=1}^{q} \sum_{\alpha_{p}=0}^{m_{p}-1} \varepsilon_{p}^{\alpha_{p} \gamma_{p}}
$$


Число $\varepsilon_{p}^{\alpha_{p} \gamma_{p}}$ является корнем многочлена $z^{m_{p}}-1$. Причем если $\gamma_{p}$ делится на $m_{p}$, то $\varepsilon_{p}^{\alpha_{p} \gamma_{p}}=1, \alpha_{p}=0,1, \ldots, m_{p}-1$. Следовательно, $\sum_{\alpha_{p}=0}^{m_{p}-1} \varepsilon_{p}^{\alpha_{p} \gamma_{p}}=m_{p}$. Если же $\gamma_{p}$ не делится на $m_{p}$, то все числа $\varepsilon_{p}^{\alpha_{p} \gamma_{p}}, \alpha_{p}=0,1, \ldots, m_{p}-1$, различны. Это означает, что совокупность $\left\{\varepsilon_{p}^{\alpha_{p} \gamma_{p}}: \alpha_{p}=0,1, \ldots, m_{p}-1\right\}$ представляет собой множество всех корней многочлена $z^{m_{p}}-1$. Понятно, что их сумма равна нулю: $\sum_{\alpha_{p}=0}^{m_{p}-1}\left(\varepsilon_{p}\right)^{\alpha_{p} \gamma_{p}}=0$. Пишем $\mu \mid \gamma$, если для всех $p \gamma_{p}$ делится на $m_{p}$, в противном случае пишем $\mu \nmid \gamma$. Таким образом,

$$
\sum_{\alpha<\mu} \varepsilon^{\alpha \gamma}=\left\{\begin{array}{ll}
m, & \text { если } \mu \mid \gamma, \\
0, & \text { если } \mu \nmid \gamma,
\end{array} \quad \pi_{\gamma}(\zeta)= \begin{cases}\zeta^{|\gamma|}, & \text { если } \mu \mid \gamma, \\
0, & \text { если } \mu \nmid \gamma .\end{cases}\right.
$$

Поэтому

$$
\begin{aligned}
f(z, h) & =\sum_{|\gamma|=0}^{\infty} \frac{\left(\pi_{\gamma}(D) f\right)(z)}{\gamma !} h^{\gamma}=\sum_{\substack{\gamma=k \mu \\
k=0,1, \ldots}} \frac{\left(D^{|\gamma|} f\right)(z)}{\gamma !} h^{\gamma} \\
& =\sum_{|\gamma|=0}^{\infty} \frac{\left(D^{|\gamma|} f\right)(z)}{\gamma !} h^{\gamma} \frac{1}{m} \sum_{\alpha<\mu} \varepsilon^{\alpha \gamma}=\frac{1}{m} \sum_{\alpha<\mu} \sum_{|\gamma|=0}^{\infty} \frac{\left(D^{|\gamma|} f\right)(z)}{\gamma !}\left(\varepsilon^{\alpha} h\right)^{\gamma}
\end{aligned}
$$

где $\left(\varepsilon^{\alpha} h\right)^{\gamma}=\left(\varepsilon_{1}^{\alpha_{1} \gamma_{1}} h_{1}^{\gamma_{1}}\right) \times \cdots \times\left(\varepsilon_{q}^{\alpha_{q} \gamma_{q}} h_{q}^{\gamma_{q}}\right)$. Зафиксируем $z \in \Omega$ и рассмотрим функцию $F\left(z_{1}, \ldots, z_{q}\right):=f\left(z_{1}+\cdots+z_{q}\right)$. Она голоморфна в некоторой окрестности точки $z^{\prime}=(\underbrace{0, \ldots, 0}_{q-1}, z)$ и при этом $\left(D^{\gamma} F\right)\left(z^{\prime}\right)=\left(D^{|\gamma|} f\right)(z)$. Значит,

$$
\begin{aligned}
f(z, h) & =\frac{1}{m} \sum_{\alpha<\mu} \sum_{|\gamma|=0}^{\infty} \frac{\left(D^{\gamma} F\right)\left(z^{\prime}\right)}{\gamma !}\left(\varepsilon^{\alpha} h\right)^{\gamma} \\
& =\frac{1}{m} \sum_{\alpha<\mu} F\left(z^{\prime}+\varepsilon^{\alpha} h\right)=\frac{1}{m} \sum_{\alpha<\mu} f\left(z+\left\langle\varepsilon^{\alpha} h\right\rangle\right),
\end{aligned}
$$

где $\varepsilon^{\alpha} h=\left(\varepsilon_{p}^{\alpha_{p}} h_{p}\right),\left\langle\varepsilon^{\alpha} h\right\rangle=\sum_{p=1}^{q} \varepsilon_{p}^{\alpha_{p}} h_{p}$. Таким образом, оператор $\pi$-сдвига превращается в оператор т-стороннего сдвига:

$$
f(z, h)=\frac{1}{m} \sum_{\alpha<\mu} f\left(z+\left\langle\varepsilon^{\alpha} h\right\rangle\right)
$$

При $m_{1}=\cdots=m_{q}=1 \pi$-сдвиг-это обычный сдвиг: $f(z, h)=f(z+h)$. Оператор $m$-стороннего сдвига введен и изучен автором в работе [13] в ситуации $q=1$.

3.4. Свойства оператора $\pi$-сдвига. Отметим некоторые свойства оператора $\pi$-сдвига $T_{h}: H(\Omega) \rightarrow H(G)(h$ фиксировано в $\mathbf{O})$.

Свойство 3.1. Для любого

$$
T_{h}\left(e^{\zeta_{0} z}\right)=e^{\zeta_{0} z}\left(\operatorname{sym}_{\lambda} e^{\langle\lambda, h\rangle}\right)\left(\zeta_{0}\right)
$$


ДокАЗАТЕЛЬство. По предложению 3.2 имеем

$$
T_{h}\left(e^{\zeta_{0} z}\right)=\left\langle\left(e^{\zeta_{0} z}\right)^{\odot}, e^{\zeta z}\left(\operatorname{sym}_{\lambda} e^{\langle\lambda, h\rangle}\right)(\zeta)\right\rangle \text {. }
$$

Учитывая $(17)$, получаем $\left(e^{\zeta_{0} z}\right)^{\odot}=\delta_{\zeta_{0}}$, где $\delta_{\zeta_{0}}-$ функционал из $P^{*}(\Omega)$, действующий по правилу $\psi \rightarrow \psi\left(\zeta_{0}\right)$. Следовательно,

$$
T_{h}\left(e^{\zeta_{0} z}\right)=\left\langle\delta_{\zeta_{0}}, e^{\zeta z}\left(\operatorname{sym}_{\lambda} e^{\langle\lambda, h\rangle}\right)(\zeta)\right\rangle=e^{\zeta_{0} z}\left(\operatorname{sym}_{\lambda} e^{\langle\lambda, h\rangle}\right)\left(\zeta_{0}\right)
$$

Тем самым свойство доказано.

Свойство 3.2. Отображение $T_{h}^{\odot}: P(G) \rightarrow P(\Omega)$, дуальное по отночению $\kappa T_{h}: H(\Omega) \rightarrow H(G)$, действует по правилу

$$
\varphi \rightarrow \varphi(\zeta)\left(\operatorname{sym}_{\lambda} e^{\langle\lambda, h\rangle}\right)(\zeta)
$$

ДоказАтЕльство. Выберем произвольную функцию $\varphi \in P(G)$. Отображение $T_{h}^{\odot}$ переводит функцию $\varphi$ в функцию $\left\langle S, T_{h}\left(e^{\zeta z}\right)\right\rangle$, где $S-$ функционал $T^{-1}(\varphi) \in$ $H^{*}(G)$. В силу $(22)\left\langle S, T_{h}\left(e^{\zeta z}\right)\right\rangle=\left\langle S, e^{\zeta z}\left(\operatorname{sym}_{\lambda} e^{\langle\lambda, h\rangle}\right)(\zeta)\right\rangle$. По определению отображения $T$ имеем $\left\langle S, e^{\zeta z}\right\rangle=\varphi(\zeta)$. Значит, $\left\langle S, T_{h}\left(e^{\zeta z}\right)\right\rangle=\varphi(\zeta)\left(\operatorname{sym}_{\lambda} e^{\langle\lambda, h\rangle}\right)(\zeta)$. Свойство доказано.

По предложению $3.2 \pi$-сдвиг $f(z, h)$ функции $f \in H(\Omega)$ на шаг $h$ как функция переменного вектора $(z, h)$ является элементом пространства $H(G \times \mathbf{O})$ и, значит, допускает дифференцирование по переменным $h_{1}, \ldots, h_{q}$. Обозначим через $D_{p}$ оператор частного дифференцирования по переменной $h_{p}$, и пусть $\pi_{p}\left(D_{p}\right) T_{h}$ - оператор, который функции $f \in H(\Omega)$ ставит в соответствие функцию $\pi_{p}\left(D_{p}\right) f(z, h)$. Здесь после дифференцирования векторная переменная $h$ фиксируется в О, т.е. функция $\pi_{p}\left(D_{p}\right) f(z, h)$ рассматривается как элемент пространства $H(G)$.

Свойство 3.3. Для любого $p=1, \ldots, q$ выполняется соотношение

$$
\pi_{p}\left(D_{p}\right) T_{h}=T_{h} \circ \pi_{p}(D) .
$$

ДоказАтельство. Достаточно установить, что совпадают дуальные операции

$$
\left(\pi_{p}\left(D_{p}\right) T_{h}\right)^{\odot}=\left(T_{h} \circ \pi_{p}(D)\right)^{\odot} .
$$

Учитывая $(18)$, получаем $\left(T_{h} \circ \pi_{p}(D)\right)^{\odot}=\pi_{p}(D) \odot \circ T_{h}^{\odot}$. В силу $(23) T_{h}^{\odot}$ - операция умножения на $\left(\operatorname{sym}_{\lambda} e^{\langle\lambda, h\rangle}\right)(\zeta)$. Легко проверить, что $\pi_{p}(D) \odot-$ операция умножения на $\pi_{p}(\zeta)$. Значит, для любой $\varphi(\zeta) \in P(G)$ выполнено

$$
\left(T_{h} \circ \pi_{p}(D)\right)^{\odot} \varphi(\zeta)=\varphi(\zeta) \pi_{p}(\zeta)\left(\operatorname{sym}_{\lambda} e^{\langle\lambda, h\rangle}\right)(\zeta) .
$$

$\mathrm{C}$ другой стороны, операция $\left(\pi_{p}\left(D_{p}\right) T_{h}\right)^{\odot}$ переводит функцию $\varphi(\zeta)=\left\langle S, e^{\zeta z}\right\rangle \in$ $P(G)$ в функцию $\left\langle S, \pi_{p}\left(D_{p}\right) T_{h}\left(e^{\zeta z}\right)\right\rangle$. В силу $(22)$ имеем

$$
\begin{aligned}
\left(\pi_{p}\left(D_{p}\right) T_{h}\right)^{\odot} \varphi(\zeta) & =\left\langle S, e^{\zeta z} \pi_{p}\left(D_{p}\right)\left(\operatorname{sym}_{\lambda} e^{\langle\lambda, h\rangle}\right)(\zeta)\right\rangle \\
& =\varphi(\zeta) \pi_{p}\left(D_{p}\right)\left(\operatorname{sym}_{\lambda} e^{\langle\lambda, h\rangle}\right)(\zeta) .
\end{aligned}
$$

При этом для всех $\zeta \in \mathbb{C}$ выполняется очевидное соотношение

$$
\pi_{p}\left(D_{p}\right) \sum_{\lambda \in\langle\zeta\rangle} e^{\langle\lambda, h\rangle}=\pi_{p}(\zeta) \sum_{\lambda \in\langle\zeta\rangle} e^{\langle\lambda, h\rangle} .
$$

Значит, $\pi_{p}\left(D_{p}\right)\left(\operatorname{sym}_{\lambda} e^{\langle\lambda, h\rangle}\right)(\zeta)=\pi_{p}(\zeta)\left(\operatorname{sym}_{\lambda} e^{\langle\lambda, h\rangle}\right)(\zeta)$. Следовательно,

$$
\left(\pi_{p}\left(D_{p}\right) T_{h}\right)^{\odot} \varphi(\zeta)=\varphi(\zeta) \pi_{p}(\zeta)\left(\operatorname{sym}_{\lambda} e^{\langle\lambda, h\rangle}\right)(\zeta) .
$$

Таким образом, из (24) и (25) вытекает выполнимость требуемого соотношения. Свойство доказано. 
Свойство 3.4. Пусть $d$ - компакт в О. Тогда семейство отображсний $T_{h}: H(\Omega) \rightarrow H(G), h \in d$, является равностепенно непрерывным.

ДокаЗАтЕльство. Достаточно установить, что семейство дуальных отображений $T_{h}^{\odot}: P(G) \rightarrow P(\Omega), \varphi \rightarrow \varphi \psi_{h}$, является равностепенно непрерывньм (п. 3.1, утверждение $\left.1^{\circ}\right)$. Здесь $\psi_{h}=\operatorname{sym}_{\lambda} e^{\langle\lambda, h\rangle}$. Известно, что операция умножения $P(G) \times P(O) \rightarrow P(\Omega),(\varphi, \psi) \rightarrow \varphi \psi$, является непрерывной [16; теорема 14.1]. Значит, для любой окрестности нуля $U \subseteq P(\Omega)$ найдутся абсолютно выпуклые окрестности нуля $V_{1} \subseteq P(G), V_{2} \subseteq P(O)$ такие, что $V_{1} V_{2} \subseteq U$. Учитывая оценку $(20)$, для любого $h=\left(h_{1}, \ldots, h_{q}\right) \in d$ имеем

$$
\left|\frac{1}{m_{p}} \sum_{\lambda_{p} \in\langle\zeta\rangle_{p}} e^{\lambda_{p} h_{p}}\right| \leqslant \frac{1}{m_{p}} \sum_{\lambda_{p} \in\langle\zeta\rangle_{p}} e^{\sigma_{d}\left|\lambda_{p}\right|} \leqslant M e^{\sigma_{1}|\zeta|},
$$

где $\sigma_{d}=\max _{h \in d} \max _{p=1, \ldots, q}\left|h_{p}\right|<\sigma_{1}<\sigma, M>0$. Значит, для любого $h \in d$ справедлива оценка $\left|\psi_{h}(\zeta)\right| \leqslant M^{q} e^{\rho_{1}|\zeta|}$, где $\rho_{1}=q \sigma_{1}<\rho$. Это означает, что множество $B=\left\{\psi_{h}: h \in d\right\}$ является ограниченным в $P(O)$. Значит, при некотором $t>0$ вьполняется включение $B \subseteq t V_{2}$. Следовательно, $V B \subseteq U$. Здесь $V=\frac{1}{t} V_{1}$. В силу произвольности выбора окрестности $U$ вложение $V B \subseteq U$ означает равностепенную непрерывность семейства отображений $T_{h}: H(\Omega) \rightarrow H(G), h \in d$. Свойство доказано.

3.5. $\pi$-свертка. Пусть $\Omega$ - односвязная область в $\mathbb{C} ; S$ - фиксированньй элемент $H^{*}(\Omega)$. Действие функционала $S$ на элементы $f \in H(\Omega)$ осуществляется по правилу:

$$
\langle S, f\rangle=\int_{K} f d \mu,
$$

где $\mu$ - некоторая комплексная мера, сосредоточенная на компакте $K \subset \Omega$. Подберем односвязную область $G$ и круговую окрестность $O$ нуля в $\mathbb{C}$ так, чтобы имели место вложения $K \subseteq G, G+O \subset \Omega$, и обозначим через О поликруговую окрестность нуля в $\mathbb{C}^{q}$, удовлетворяющую условию $(21)$.

Пусть $f \in H(\Omega)$. По предложению 3.2 функция $f(z, h)$ будет аналитической на множестве $G \times \mathbf{O}$. Поэтому имеет смысл выражение

$$
(S * f)(h):=\int_{K} f(z, h) d \mu .
$$

Это выражение представляет собой функцию $h$, голоморфную в $\mathbf{O}$, и называется $\pi$-сверткой функционала $S$ и функиии $f$. Если $\pi(\zeta)=\left(\zeta_{1}^{m_{1}}, \ldots, \zeta_{1}^{m_{q}}\right)$, $\mu=\left(m_{1}, \ldots, m_{p}\right)$ - фиксированный вектор из $\mathbb{Z}_{+}^{q}$ с ненулевыми компонентами, то $\pi$-свертка преврашается в $m$-стороннюю свертку

$$
(S * f)(h)=\frac{1}{m} \sum_{\alpha<\mu}\left\langle S, f\left(z+\left\langle\varepsilon^{\alpha} h\right\rangle\right)\right\rangle,
$$

а при $\pi=\left(\zeta_{1}, \ldots, \zeta_{n}\right)$ - в обычную свертку $(S * f)(h)=\langle S, f(z+h)\rangle$.

Обозначим через $u$ оператор $\pi$-свертки $f \rightarrow S * f$, действующий из пространства $H(\Omega)$ в пространство $H(\mathbf{O})$. Исследуем свойства оператора $\pi$-свертки. 
Свойство 3.5. Oператор $u: H(\Omega) \rightarrow H(\mathbf{O})$ есть линейное непрерывное отображсние.

ДокАЗАТЕЛьство. Линейность оператора $\pi$-свертки вытекает из линейности оператора $\pi$-сдвига. Докажем непрерывность оператора $\pi$-свертки. Множества вида $V_{d, \varepsilon}=\left\{f \in H(\mathbf{O}): \max _{d}|f| \leqslant \varepsilon\right\}$, где $\varepsilon>0, d$ - компакт в $\mathbf{O}$, образуют фундаментальную систему окрестностей нуля в $H(\mathbf{O})$. Поэтому достаточно доказать следуюшее утверждение: для любого $\varepsilon>0$ и любого компакта $d \subset \mathbf{O}$ существует окрестность нуля $U \subseteq H(\Omega)$ такая, что $u(U) \subseteq V_{d, \varepsilon}$. Мера $\mu$, определяющая функционал $S$, сосредоточена на компакте $K \subset \Omega$. Пусть

$$
V_{K}=\left\{f \in H(\Omega): \sup _{K}|f| \leqslant 1\right\}, \quad\|S\|=\sup \left\{|\langle S, f\rangle|: f \in V_{K}\right\} .
$$

Можно предполагать, что $\|S\|>0$. Семейство операторов $\pi$-сдвига $T_{h}: H(\Omega) \rightarrow$ $H(G), h \in d$, является равностепенно непрерьвньп (свойство 3.4 ). Поэтому сушествует окрестность нуля $U \subseteq H(\Omega)$ такая, что $T_{h}(U) \subseteq \frac{\varepsilon}{\|S\|} V_{K} \forall h \in d$, т.е. для $f \in U$ и $h \in d$ имеем $\sup _{z \in K}|f(z, h)| \leqslant \frac{\varepsilon}{\|S\|}$. Значит,

$$
|(S * f)(h)| \leqslant\|S\| \sup _{z \in K}|f(z, h)| \leqslant \varepsilon
$$

Отсюда следует, что $\max _{d}|(S * f)| \leqslant \varepsilon$. Последнее означает, что $u(f) \in V_{d, \varepsilon}$. Это верно для любой $f \in U$. Значит, $u(U) \subseteq V_{d, \varepsilon}$. Свойство доказано.

Пусть $D_{p}$ - оператор частного дифференцирования по переменной $h_{p}$.

Свойство 3.6. Для любого $f \in H(\Omega)$ имеет место соотношение

$$
\pi_{p}\left(D_{p}\right)(S * f)=S *\left(\pi_{p}(D) f\right) .
$$

ДокАЗАТЕЛьСтво. Из линейности и непрерывности функционала $S$ вытекает

$$
\pi_{p}\left(D_{p}\right)\left\langle S, T_{h}(f)\right\rangle=\left\langle S, \pi_{p}\left(D_{p}\right) T_{h}(f)\right\rangle
$$

В силу свойства 3.3 оператора $\pi$-сдвига $\pi_{p}\left(D_{p}\right) T_{h}(f)=T_{h}\left(\pi_{p}(D) f\right)$. Значит,

$$
\begin{aligned}
\pi_{p}\left(D_{p}\right)(S * f) & =\pi_{p}\left(D_{p}\right)\left\langle S, T_{h}(f)\right\rangle=\left\langle S, \pi_{p}\left(D_{p}\right) T_{h}(f)\right\rangle \\
& =\left\langle S, T_{h}\left(\pi_{p}(D) f\right)\right\rangle=S *\left(\pi_{p}(D) f\right) .
\end{aligned}
$$

Таким образом, свойство доказано.

Свойство 3.7. Дуальная операция $u^{\odot}: P(\mathbf{O}) \rightarrow P(\Omega)$ имеет вид $\phi \rightarrow$ $\varphi \operatorname{sym} \phi$, где $\varphi-$ преобразование Лапласа функционала $S$.

ДокАзАтельство. Дуальная операция переводит функцию $\phi(\lambda)=\left\langle s, e^{\langle\lambda, h\rangle}\right\rangle$ в функцию $\psi(\zeta)=\left\langle s,\left\langle S, T_{h}\left(e^{\zeta z}\right)\right\rangle\right\rangle$. По свойству 3.1 оператора $\pi$-сдвига имеем

$$
\psi(\zeta)=\left\langle s,\left\langle S,\left(e^{\zeta z} \operatorname{sym}_{\lambda} e^{\langle\lambda, h\rangle}\right)(\zeta)\right\rangle\right\rangle=\varphi(\zeta)\left\langle s,\left(\operatorname{sym}_{\lambda} e^{\langle\lambda, h\rangle}\right)(\zeta)\right\rangle
$$

При этом очевидно, что

$$
\left\langle s,\left(\operatorname{sym}_{\lambda} e^{\langle\lambda, h\rangle}\right)(\zeta)\right\rangle=\left(\operatorname{sym}_{\lambda}\left\langle s, e^{\langle\lambda, h\rangle}\right\rangle\right)(\zeta)=\left(\operatorname{sym}_{\lambda} \phi(\lambda)\right)(\zeta) .
$$

Значит, $\psi=\varphi \operatorname{sym} \phi$. Тем самым свойство доказано. 
3.6. Однородное уравнение $\pi$-свертки. Пусть $\Omega=\left\{\Omega_{1}, \ldots, \Omega_{\nu}\right\}$ - некоторая система односвязных областей в $\mathbb{C} ; H_{j}=H\left(\Omega_{j}\right)$ - пространство функций, голоморфных в $\Omega_{j}$, с топологией компактной сходимости; $\mathbf{H}$ - топологическое произведение $H_{1} \times \cdots \times H_{\nu} ; S=\left(S_{1}, \ldots, S_{\nu}\right)$ - фиксированный элемент $\mathbf{H}^{*}$. Действие функционала $S$ на элементы $f=\left(f_{1}, \ldots, f_{\nu}\right) \in \mathbf{H}$ осуществляется по правилу:

$$
\langle S, f\rangle=\sum_{j=1}^{\nu}\left\langle S_{j}, f_{j}\right\rangle=\sum_{j=1}^{\nu} \int_{K_{j}} f_{j} d \mu_{j},
$$

где $\mu_{j}$ - некоторая комплексная мера, сосредоточенная на компакте $K_{j} \subset \Omega_{j}$. Подберем односвязные области $G_{j}$ и круговую окрестность $O$ нуля так, чтобы имели место вложения $K_{j} \subset G_{j}, G_{j}+O \subset \Omega_{j}$, и обозначим через О поликруговую окрестность нуля в $\mathbb{C}^{q}$, удовлетворяющую условию $(21)$ :

$$
\left\{\zeta: \zeta=\lambda_{1}+\cdots+\lambda_{q}, \lambda=\left(\lambda_{1}, \ldots, \lambda_{q}\right) \in \mathbf{O}\right\} \subseteq O
$$

По предложению $3.2 \pi$-сдвиги $f_{1}(z, h), \ldots, f_{\nu}(z, h)$ будут аналитическими на множествах $G_{1} \times \mathbf{O}, \ldots, G_{\nu} \times \mathbf{O}$ соответственно. Поэтому имеет смысл выражение

$$
S * f:=\sum_{j=1}^{\nu} \int_{K_{j}} f_{j}(z, h) d \mu_{j} .
$$

Выражение $S * f$ представляет собой функцию $h$, голоморфную в $\mathbf{O}$, и называется $\pi$-сверткой функционала $S$ и әлемента $f$. Рассмотрим однородное уравнение л-свертки

$$
S * f=0, \quad f \in \mathbf{H} .
$$

ПРЕДЛОЖЕНИЕ 3.3. Множсество $W$ решений $f \in \mathbf{H}$ однородного уравнения (26) есть замкнутое инвариантное подпространство в $\mathbf{H}$.

ДокАЗАТЕЛЬство. То, что $W$ - замкнутое подпространство Н, следует из линейности и непрерьвности оператора $\pi$-свертки (свойство 3.5$)$. Далее, пусть $f \in$ $W$, значит, $(S * f)(h) \equiv 0$. Действуя оператором $\pi_{p}\left(D_{p}\right)$ и используя свойство 3.6 оператора $\pi$-свертки, получаем $\pi_{p}\left(D_{p}\right)(S * f)(h) \equiv\left(S * \pi_{p}(D) f\right)(h) \equiv 0$. Это значит, что $\pi_{p}(D) f \in W$. Таким образом, $f \in W \Rightarrow \pi_{p}(D) f \in W, p=1, \ldots, q$. Предложение доказано.

Корневые элементы системы операторов (1), удовлетворяющие уравнению (26), называются әлементарными решениями этого уравнения. Задача спектрального синтеза для подпространства $W$ решений этого уравнения принимает форму классической задачи: каждое ли решение уравнения (26) можно аппроксимировать в Н линейными комбинациями әлементарных решений?

Следующее предложение раскрывает строение аннуляторного подмодуля подпространства $W$.

ПРЕДЛОЖЕНИЕ 3.4. Аннуляторный подмодуль I инвариантного подпространства решений однородного уравнения $\pi$-свертки (26) есть главный $\mathbb{C}[\pi]-$ подмодуль в $\mathbf{P}$, порожсденный әлементом $\varphi=\mathbf{T}(S)$. 
ДокАЗАТЕЛЬСТво. Подпространство $W$ решений уравнения (26) есть ядро оператора $\pi$-свертки $u: \mathbf{H} \rightarrow H(\mathbf{O}), f=\left(f_{j}\right) \rightarrow \sum_{j=1}^{\nu} u_{j}\left(f_{j}\right)$, где $u_{j}: H_{j} \rightarrow H(\mathbf{O})-$ оператор $\pi_{j}$-свертки. Оператор $u$ является непрерывным. Так как пространство $\mathbf{H}$ является рефлексивньп, то ортогональное подпространство $W^{0} \subseteq \mathbf{H}^{*}$ совпадает с замыканием в $\mathbf{H}^{*}$ образа $u^{*}\left(H^{*}(\mathbf{O})\right)[21 ; 8.6]$. Последнее равносильно тому, что $I=\mathbf{T}\left(W^{0}\right)$ совпадает с замьканием в $\mathbf{P}$ образа $u^{\odot}(P(\mathbf{O}))$, где $u^{\odot}-$ дуальная к $u$ операция. Легко убедиться, что сопряженная операция $u^{*}: H^{*}(\mathbf{O}) \rightarrow \mathbf{H}^{*}$ действует по правилу $s \rightarrow\left(u_{1}^{*}(s), \ldots, u_{\nu}^{*}(s)\right)$, а дуальная операция $u^{\odot}: P(\mathbf{O}) \rightarrow \mathbf{P}$ - по правилу $\phi \rightarrow\left(u_{1}^{\odot}(\phi), \ldots, u_{\nu}^{\odot}(\phi)\right)$. Значит, $I$ есть замыкание в $\mathbf{P}$ множества элементов вида $\varphi \operatorname{sym} \phi$, где $\phi \in P(\mathbf{O})$ (см. свойство 3.7). Путем элементарных оценок легко убедиться, что частичные суммы $\sum_{|\gamma|=0}^{n} c_{\gamma} \lambda^{\gamma}$ ряда Тейлора функции $\phi$ сходятся к ней в топологии $P(\mathbf{O})$ (см., например, $[22 ; \S 12])$. По предложению $3.1 r_{n}=\sum_{|\gamma|=0}^{n} c_{\gamma} \pi_{\gamma} \rightarrow \operatorname{sym} \phi$ в топологии $P(O)$. Билинейная операция $P\left(G_{j}\right) \times P(O) \rightarrow P\left(\Omega_{j}\right),\left(\phi_{j}, \psi\right) \rightarrow \phi_{j} \psi$, является раздельно непрерывной $[16 ;$ теорема 14.1]. Значит, $\varphi_{j} r_{n} \rightarrow \varphi_{j} \operatorname{sym} \phi$ в топологии $P\left(\Omega_{j}\right)$. Поэтому элементы вида $\varphi$ sym $\phi$ можно аппроксимировать в топологии $\mathbf{P}$ элементами вида $\varphi r$, где $r \in \operatorname{sym} \mathbb{C}[\lambda]=\mathbb{C}[\pi]$. Это и требовалось доказать.

СлеДСТВИЕ 3.1. Аннуляторный подмодуль инвариантного подпространства решений системы однородных уравнений $\pi$-свертки

$$
\left\{\begin{array}{l}
S^{(1)} * f=0, \\
\ldots \ldots \ldots \ldots \\
S^{(k)} * f=0,
\end{array} \quad f \in \mathbf{H},\right.
$$

есть конечно порожденный $\mathbb{C}[\pi]$-подмодуль в $\mathbf{P}$, порожденный әлементами $\varphi^{(1)}=\mathbf{T}\left(S^{(1)}\right), \ldots, \varphi^{(k)}=\mathbf{T}\left(S^{(k)}\right)$.

ДокАЗАТЕЛЬСтво. Пусть $W^{(1)}, \ldots, W^{(k)}$-подпространства решений однородных уравнений $\pi$-свертки $S^{(1)} * f=0, \ldots, S^{(k)} * f=0$ соответственно. Тогда пересечение $W=W^{(1)} \cap \cdots \cap W^{(k)}$ является подпространством решений системы (27). По свойствам поляр аннулятор $V=W^{0} \subset \mathbf{H}^{*}$ совпадает с замыканием в $\mathbf{H}^{*}$ множества $V^{(1)}+\cdots+V^{(k)}$, где $V^{(i)}$ - аннулятор подпространства $W^{(i)}$, $i=1, \ldots, k$. Значит, аннуляторный подмодуль $I=$ An $W$ совпадает с замыканием в $\mathbf{P}$ множества $I^{(1)}+\cdots+I^{(k)}$, где $I^{(i)}$ - аннуляторньй подмодуль подпространства $W^{(i)}, i=1, \ldots, k$. Таким образом, любой элемент из $I$ можно аппроксимировать в топологии $\mathbf{P}$ суммами $f^{(1)}+\cdots+f^{(k)}, f^{(i)} \in I^{(i)}$. Из предложения 3.4 вытекает, что любой элемент из $I$ можно аппроксимировать элементами вида $r^{(1)} \varphi^{(1)}+\cdots+r^{(k)} \varphi^{(k)}, r^{(i)} \in \mathbb{C}[\pi]$. Согласно определению это означает, что $I-\mathbb{C}[\pi]$-подмодуль в $\mathbf{P}$, порожденный совокупностью элементов $\varphi^{(1)}, \ldots, \varphi^{(k)}$. Следствие доказано.

\section{§4. Спектральный синтез}

4.1. Основные следствия критерия обильности. Выберем произвольное замкнутое $\pi(D)$-инвариантное подпространство $W \subseteq \mathbf{H}$ и обозначим через $I$ его аннуляторньй подмодуль. Из теоремы двойственности (см. п. 1.3) вытекает, что допустимость спектрального синтеза подпространством $W$ эквивалентна обильности $I$. Пусть $\Omega_{1}, \ldots, \Omega_{\nu}$ - выпуклые области. Тогда по критерию обильности 
(см. п. 2.3) свойство обильности замкнутого $\mathbb{C}[\pi]$-подмодуля в $\mathbf{P}$ расщепляется на два свойства - устойчивость и насыщенность. Таким образом, справедлив следующий критерий.

ТеОРема 4.1. Замкнутое $\pi(D)$-инвариантное подпространство $W \subseteq \mathbf{H}$ допускает спектральный синтез тогда и только тогда, когда его аннуляторный подмодуль $I \subseteq \mathbf{P}$ устойчив и насъщен.

Теорема 4.1 сводит исследование замкнутого $\pi(D)$-инвариантного подпространства на предмет допустимости им спектрального синтеза к проверке устойчивости и насышенности его аннуляторного подмодуля. Следующая теорема посвящена ситуации, в которой эту проверку можно упростить.

ТЕОРема 4.2. Пусть $W$ - замкнутое $\pi(D)$-инвариантное подпространство $\mathbf{H}$, аннуляторный подмодуль $I \subseteq \mathbf{P}$ которого имеет $\pi$-ранг равный единиие. Для того чтобы подпространство $W$ допускало спектральный синтез, необходимо и достаточно, чтобъ подмодуль I был устойчив.

ДокАЗАТЕЛЬство. Необходимость. Пусть $W$ - замкнутое $\pi(D)$-инвариантное подпространство Н, допускаюшее спектральный синтез. По теореме двойственности аннуляторный подмодуль $I$ подпространства $W$ является обильным. По критерию обильности подмодуль $I$ является устойчивым. Необходимость доказана.

Достаточность. Допустим, что аннуляторньй подмодуль $I$ подпространства $W \subseteq \mathbf{H}$ является устойчивым и имеет $\pi$-ранг 1 . В силу теоремы двойственности нам достаточно доказать, что подмодуль $I$ является обильньм. Непосредственная проверка показывает, что $\mathbb{C}[\pi]$-подмодули в $\mathbf{P} \pi$-ранга 1 автоматически являются насышенными $[11 ; \S 3$, п. 3.5]. Значит, по критерию обильности устойчивые подмодули $\pi$-ранга 1 являются обильньми. Но легче доказать обильность подмодуля $I$, опираясь на теорему редукции (см. п. 1.4) и теорему 3.2 из [11]. Пусть $l-$ многочлен Люрота системы многочленов $\pi_{1}, \ldots, \pi_{q}$. По следствию 2.3 замкнутый $\mathbb{C}[l]$-подмодуль $J \subseteq \mathbf{P}$, порождаемый $I$, имеет $l$-ранг 1 . По теореме 2.1 подмодуль $J$ устойчив. Следовательно, по теореме 3.2 из [11] подмодуль $J$ является обильным. В то же время, по теореме редукции обильность подмодуля $J$ влечет обильность подмодуля $I$. Теорема доказана.

Из теоремы 4.2 вытекает, что теория спектрального синтеза для системы дифференциальных операторов $\pi_{1}(D), \ldots, \pi_{q}(D)$ в случае $\pi$-Rank $I=1$ повторяет теорию спектрального синтеза для обычного оператора дифференцирования.

Рассмотрим два следствия теоремы 4.2.

СЛЕДСТВИЕ 4.1. Пусть система $\varphi^{(1)}, \ldots, \varphi^{(k)}$ элементов из $\mathbf{P}$ имеет $\pi$-ранг равный единице. Замкнутое $\pi(D)$-инвариантное подпространство $W \subseteq \mathbf{H}$ решений системы однородных уравнений $\pi$-свертки (27) допускает спектральный синтез тогда и только тогда, когда его аннуляторный подмодуль $I \subseteq \mathbf{P}$ устойчив.

ДокАЗАТЕЛЬСтво. По предложению 3.4 (см. следствие 3.1 ) аннуляторный подмодуль $I \subseteq \mathbf{P}$ замкнутого $\pi(D)$-инвариантного подпространства $W \subseteq \mathbf{H}$ решений системы однородных уравнений $\pi$-свертки (27) является конечно порожденным $\mathbb{C}[\pi]$-подмодулем в $\mathbf{P}$ с образуюшими $\varphi^{(1)}, \ldots, \varphi^{(k)}$. Так как эта система элементов из $\mathbf{P}$ имеет $\pi$-ранг 1 , то по следствию $2.3 \pi$-Rank $I=1$. Осталось сослаться на теорему 4.2. Следствие доказано. 
Пусть $\Omega$ - выпуклая область в $\mathbb{C} ; m_{1}=\operatorname{deg} \pi_{1}, \ldots, m_{q}=\operatorname{deg} \pi_{q}$. Если наибольший общий делитель (НОД) чисел $m_{1}, \ldots, m_{q}$ равен единище, то справедливо следуюшее утверждение.

СЛЕДСТВИЕ 4.2. Замкнутое $\pi(D)$-инвариантное подпространство $W \subseteq$ $H(\Omega)$ допускает спектральный синтез тогда и только тогда, когда его аннуляторный подмодуль $I \subseteq P(\Omega)$ устойчив.

ДокАЗАТЕЛЬСТво. Пусть $W$ - замкнутое $\pi(D)$-инвариантное подпространство $H(\Omega), I \subseteq P(\Omega)$ - его аннуляторный подмодуль. Достаточно показать, что при условии НОД $\left(m_{1}, \ldots, m_{q}\right)=1$ выполняется равенство $\pi$-Rank $I=1$. Пусть $l-$ многочлен Люрота для системы многочленов $\pi_{1}, \ldots, \pi_{q}$. В силу правого включения из $(3)$ степень $m$ многочлена $l$ является делителем чисел $m_{1}, \ldots, m_{q}$. Поэтому если НОД $\left(m_{1}, \ldots, m_{q}\right)=1$, то $m=1$ и, значит, по следствию 2.2 , учитьвая, что $\nu=1$, имеем $\pi$-Rank $I=1$. Тем самым следствие доказано.

С другой стороны, по предложению $2.3 \mathbb{C}[\pi]$-подмодуль $I \subseteq \mathbf{P}$, порожденньй $\pi$-независимой системой элементов, всегда является устойчивым. В силу теоремы 4.1 справедливо следующее предложение.

ПрЕДЛОЖЕНИЕ 4.1. Пусть система $\varphi^{(1)}, \ldots, \varphi^{(k)}$ әлементов из $\mathbf{P}$ является $\pi$-независимой. Замкнутое $\pi(D)$-инвариантное подпространство $W \subseteq \mathbf{H}$ решений системы однородных уравнений т-свертки (27) допускает спектральный синтез тогда и только тогда, когда его аннуляторный подмодуль $I \subseteq \mathbf{P}$ насыщен.

Пусть $I^{(1)}, \ldots, I^{(k)}$ - главные $\mathbb{C}[\pi]$-подмодули в $\mathbf{P}$, порождаемые элементами $\varphi^{(1)}, \ldots, \varphi^{(k)}$ соответственно. Зафиксируем простой $l$-слой $\tilde{\xi}$, не пересекающийся с нулевьм множеством многочлена $s$, для которого

$$
l-\operatorname{Ind}_{\widetilde{\xi}} I^{(1)}=\cdots=l-\operatorname{Ind}_{\widetilde{\xi}} I^{(k)}=0 .
$$

Положим

$$
A(\widetilde{\xi})=\left\{a=\left(a_{1}, \ldots, a_{k}\right) \in \mathbb{C}^{k}: a_{1} \varphi^{(1)}(\omega)+\cdots+a_{k} \varphi^{(k)}(\omega)=0 \forall \omega \in \widetilde{\xi}\right\} .
$$

Комбинируя предложение 2.5, предложение 3.4 и теорему 4.1, получаем следуюший результат.

ПРЕДЛОЖЕНИЕ 4.2. Пусть для кажсдого вектора $а \in A(\widetilde{\xi})$ существуют обобщенные последовательности $\left\{p_{1 \sigma}\right\}, \ldots,\left\{p_{k \sigma}\right\}, \sigma \in \Sigma$, многочленов из кольиа $\mathbb{C}[l]$ такие, что:

1) $p_{1 \sigma}(\widetilde{\xi})=a_{1}, \ldots, p_{k \sigma}(\widetilde{\xi})=a_{k}$;

2) $p_{1 \sigma} \varphi^{(1)}+\cdots+p_{k \sigma} \varphi^{(k)} \rightarrow 0$ в топологии $\mathbf{P}$.

Тогда для того чтобъ подпространство $W \subseteq \mathbf{H}$ решений системь однородных уравнений свертки (26) допускало спектральный синтез, необходимо и достаточно, чтобъ аннуляторный подмодуль I подпространства W бъл насышен.

Пусть $W^{(1)}, \ldots, W^{(k)}$ - замкнутые $\pi(D)$-инвариантные подпространства $\mathbf{H}$; $I^{(1)}, \ldots, I^{(k)}$ - их аннуляторные подмодули. Аннуляторньй подмодуль $I$ замкнутого $\pi(D)$-инвариантного подпространства $W=W^{(1)} \cap \cdots \cap W^{k} \subseteq \mathbf{H}$ совпадает 
с суммой подмодулей $I^{(1)}, \ldots, I^{(k)}$. Выберем простой $l$-слой $\widetilde{\xi}$, не пересекающийся с нулевым множеством многочлена $s$, для которого имеют место соотношения (28). Рассмотрим набор $\alpha=\left(\alpha^{(1)}, \ldots, \alpha^{(k)}\right)$ функций $\widetilde{\xi} \rightarrow \mathbb{C}^{\nu}$, допустимых относительно подмодулей $I^{(1)}, \ldots, I^{(k)}$ соответственно (см. п. 2.1). Положим

$$
\begin{aligned}
T(I, \alpha, \widetilde{\xi})=\{\varphi & =\varphi^{(1)}+\cdots+\varphi^{(k)}: \varphi^{(i)} \in I^{(i)} \\
& \left.\varphi^{(i)}(\omega)=\alpha^{(i)}(\omega) \forall \omega \in \widetilde{\xi}, i=1, \ldots, k\right\} .
\end{aligned}
$$

Комбинация предложения 2.4 и теоремы 4.1 приводит к следующему результату.

ПРЕДЛОЖЕНИЕ 4.3. Пусть $I^{(1)}, \ldots, I^{(k)}$ - устойчивые $\mathbb{C}[\pi]$-подмодули в $\mathbf{P}$ и для любого набора $\alpha^{(1)}, \ldots, \alpha^{(k)}, \alpha^{(1)}+\cdots+\alpha^{(k)}=0$, функций $\alpha^{(i)}: \widetilde{\xi} \rightarrow \mathbb{C}^{\nu}$, допустимых относительно $I^{(1)}, \ldots, I^{(k)}$ соответственно, замыкание множества $T(I, \alpha, \widetilde{\xi})$ в топологии $\mathbf{P}$ содержит нулевой әлемент. Тогда для того чтобъ пересечение $W=W^{(1)} \cap \cdots \cap W^{(k)}$ допускало спектральный синтез, необходимо и достаточно, чтобь сумма подмодулей $I^{(1)}, \ldots, I^{(k)}$ бъла насышена.

4.2. Двойственный эквивалент теоремы редукции. Пусть $l$-многочлен Люрота системы многочленов $\pi_{1}, \ldots, \pi_{q} ; l(D)$ - соответствующий дифференциальный оператор с постоянными коэффициентами, действующий в Н покомпонентно. Теорема редукции допускает двойственную интерпретацию в терминах задачи спектрального синтеза для систем дифференциальных операторов.

Выберем произвольное замкнутое подпространство $W_{0}$ в Н. Обозначим через $W$ замкнутое $\pi(D)$-инвариантное подпространство в $\mathbf{H}$, содержашееся в $W_{0}$ и обладающее свойством максимальности: всякое замкнутое $\pi(D)$-инвариантное подпространство в $\mathbf{H}$, вложенное в $W_{0}$, вложено в $W$. Аналогично, обозначим через $V$ замкнутое $l(D)$-инвариантное подпространство в $\mathbf{H}$, содержашееся в $W_{0}$ и обладающее свойством максимальности: всякое замкнутое $l(D)$-инвариантное подпространство в $\mathbf{H}$, вложенное в $W_{0}$, вложено в $V$.

ТЕОРема 4.3. Замкнутое $\pi(D)$-инвариантное подпространство $W$ допускает спектральный синтез тогда и только тогда, когда замкнутое $l(D)$-инвариантное подпространство $V$ допускает спектральный синтез.

ДоказАтельство. Пусть $I_{0}=\operatorname{An} W_{0}, I=\operatorname{An} W, J=\operatorname{An} V$. Аннуляторный подмодуль $I$ включает множество $I_{0}$ и обладает свойством минимальности: всякий замкнутый $\mathbb{C}[\pi]$-подмодуль в $\mathbf{P}$, включающий $I_{0}$, включает $I$. Действительно, пусть $I^{\prime}$ - произвольный замкнутый $\mathbb{C}[\pi]$-подмодуль в $\mathbf{P}$, включающий $I_{0} ; W^{\prime}-$ $\pi(D)$-инвариантное подпространство в $\mathbf{H}$, аннуляторньй подмодуль которого совпадает с $I^{\prime}$. Из включения $I_{0} \subseteq I^{\prime}$ вытекает, что подпространство $W^{\prime}$ является подмножеством $W_{0}$. В силу свойства максимальности $W$ выполняется включение $W^{\prime} \subseteq W$. Следовательно, $I \subseteq I^{\prime}$. Таким образом, свойство минимальности подмодуля $I$ доказано. С другой стороны, минимальный замкнутый $\mathbb{C}[\pi]$-подмодуль в $\mathbf{P}$, включающий множество $I_{0}$, совпадает с замкнутьм $\mathbb{C}[\pi]$-подмодулем $\overline{I_{0}[\pi]} \subseteq \mathbf{P}$, порождаемьм множеством $I_{0}$. Значит, $I=\overline{I_{0}[\pi]}$. Аналогичным образом доказывается соотношение $J=\overline{I_{0}[l]}$. Здесь $\overline{I_{0}[l]}$ - замкнутый $\mathbb{C}[l]$-подмодуль в $\mathbf{P}$, порождаемый множеством $I_{0}$. По теореме редукции подмодули $I$ и $J$ обильны или нет 
одновременно. В силу теоремы двойственности подпространства $W$ и $V$ допускают спектральный синтез или нет одновременно. Тем самым теорема доказана.

4.3. Основные следствия теоремы редукции. Пусть $S^{(1)}, \ldots, S^{(k)}$ - фиксированные элементы $\mathbf{H}^{*} ; W$ - подпространство решений системы однородных уравнений $\pi$-свертки (27); $V$ - подпространство решений системы однородных уравнений $l$-свертки

$$
\left\{\begin{array}{l}
S^{(1)} \stackrel{(l)}{*} f=0, \\
\cdots \ldots \ldots \ldots \\
S^{(k)} \stackrel{(l)}{*} f=0,
\end{array} \quad f \in \mathbf{H} .\right.
$$

По предложению 3.4 (см. следствие 3.1 ) аннуляторный подмодуль $I=\mathbf{T}\left(W^{0}\right)$ - замкнутый конечно порожденный $\mathbb{C}[\pi]$-подмодуль в $\mathbf{P}$ с образующими $\varphi^{(1)}, \ldots, \varphi^{(k)}$; аннуляторный подмодуль $J=\mathbf{T}\left(V^{0}\right)$ - замкнутый конечно порожденный $\mathbb{C}[l]$-подмодуль в $\mathbf{P}$ с теми же образующими. По теореме редукции подмодули $I, J$ обильны или нет одновременно. В силу теоремы двойственности справедлива

ТЕОРЕма 4.4. Каждое решение системы однородных уравнений $\pi$-свертки (27) можсно аппроксимировать линейными комбинациями элементарных решений тогда и только тогда, когда аналогичную аппроксимацию допускает каждое решение системы однородных уравнений l-свертки (29).

В работе [19] доказано, что замкнутое $l(D)$-инвариантное подпространство решений одного однородного уравнения $l$-свертки всегда допускает спектральный синтез. Значит, из теоремы 4.4 вытекает

ПРЕДЛОЖЕНИЕ 4.4. Каждое решение однородного уравнения $\pi$-свертки можсно аппроксимировать линейными комбиначиями элементарных решений.

Теорема 4.4 имеет еще одно важное следствие в случае $l(\zeta)=\zeta^{m}, m \in \mathbb{N}$. Элемент $\varphi \in \mathbf{P}$ делит элемент $\Phi \in \mathbf{P}$ в классе целых $l$-симметричных функций, если существует целая $l$-симметричная функция $f$ такая, что $f \varphi=\Phi$. Привлекая теорему 7.5 из [13], получаем

ПРЕДЛОЖЕНИЕ 4.5. Пусть $l(\zeta)=\zeta^{m}, m \in \mathbb{N}, u$ каждый әлемент $\varphi^{(1)}, \ldots$, $\varphi^{(k)} \in \mathbf{P}$ делит некоторый әлемент $\Phi \in \mathbf{P}, \Phi \neq 0$, в классе иельх l-симметричных функиий. Тогда каждое решение системы однородных уравнений $\pi$-свертки (27) можсно аппроксимировать линейными комбинациями элементарных решений.

Отметим, что условие $l(\zeta)=\zeta^{m}, m \in \mathbb{N}$, выполняется, если кольцо $\mathbb{C}[\pi]$ содержит хотя бы один одночлен, например хотя бы один из многочленов $\pi_{1}, \ldots, \pi_{q}$ является одночленом. Убедимся в этом. Пусть $\pi_{1}(\zeta)=\zeta^{m_{1}}, m_{1} \in \mathbb{N}$. Так как $\mathbb{C}[\pi] \subseteq \mathbb{C}[l]$, то $\zeta^{m_{1}} \in \mathbb{C}[l]$. Следовательно, найдется многочлен $p$ такой, что $\zeta^{m_{1}}=p(l(\zeta))$. Если $l$ - многочлен Люрота, то $a l+b, a, b \in \mathbb{C}, a \neq 0$, - тоже многочлен Люрота. Значит, мы вправе считать, что старший коэффициент многочлена $l(\zeta)$ равен единице и $l(0)=0$. Следовательно, $p(0)=0$. Отсюда вытекает, что любой корень многочлена $l(\zeta)$ является корнем одночлена $\zeta^{m_{1}}$. Значит, $l(\zeta)-$ одночлен вида $\zeta^{m}, m \in \mathbb{N}$. 
Рассмотрим еще одну важную ситуацию: $\pi_{1}(\zeta)=\zeta^{m_{1}}, \ldots, \pi_{q}(\zeta)=\zeta^{m_{q}}, m_{i} \in \mathbb{N}$. В этом случае $l(\zeta)=\zeta^{m}$, где $m=\operatorname{HОД}\left(m_{1}, \ldots, m_{q}\right)$. Действительно, пусть $\mathbb{C}\left(\zeta^{m_{1}}, \ldots, \zeta^{m_{q}}\right)$ - поле частных кольца $\mathbb{C}\left[\zeta^{m_{1}}, \ldots, \zeta^{m_{q}}\right], \mathbb{C}\left(\zeta^{m}\right)-$ поле частных кольца $\mathbb{C}\left[\zeta^{m}\right]$. По определению элемента Люрота нам достаточно показать, что выполняется равенство $\mathbb{C}\left(\zeta^{m_{1}}, \ldots, \zeta^{m_{q}}\right)=\mathbb{C}\left(\zeta^{m}\right)$. Вложение $\mathbb{C}\left(\zeta^{m_{1}}, \ldots, \zeta^{m_{q}}\right) \subseteq$ $\mathbb{C}\left(\zeta^{m}\right)$ очевидно, так как каждьй из одночленов $\zeta^{m_{1}}, \ldots, \zeta^{m_{q}}$ является в то же время одночленом от $\zeta^{m}$. С другой стороны, известно, что наибольший общий делитель $m$ чисел $m_{1}, \ldots, m_{q}$ представляется в виде $m=n_{1} m_{1}+\cdots+n_{q} m_{q}$, где $n_{1}, \ldots, n_{q} \in \mathbb{Z}$. Отсюда вытекает, что $\zeta^{m}=\left(\zeta^{m_{1}}\right)^{n_{1}} \times \cdots \times\left(\zeta^{m_{q}}\right)^{n_{q}}$. Это означает, что одночлен $\zeta^{m}$ является рациональной функцией от $\zeta^{m_{1}}, \ldots, \zeta^{m_{q}}$. Следовательно, $\mathbb{C}\left(\zeta^{m}\right) \subseteq \mathbb{C}\left(\zeta^{m_{1}}, \ldots, \zeta^{m_{q}}\right)$.

Пусть $\Omega_{1}=\mathbb{C}, \ldots, \Omega_{\nu}=\mathbb{C}, \mathbf{H}$ - топологическое произведение $H(\mathbb{C}) \times \cdots \times H(\mathbb{C})$ $\nu$ копий пространства $H(\mathbb{C})$. Из теоремы 4.3 и [19; теорема 3.7] вытекает

ПРЕДЛОЖЕНИЕ 4.6. Всякое замкнутое $\pi(D)$-инвариантное подпространство $W \subseteq \mathbf{H}$ допускает спектральный синтез.

Для случая $\nu=1, q=1, \pi(D)=D$ этот результат получен Л. Шварцем [23], для случая $\nu=1, q=1, \pi(D)=D^{m}-$ С. Г. Мерзляковым [24]. Случай $q=1$ рассмотрен И.. Красичковьм-Терновским [15].

Если $\nu=1, l(\zeta)=\zeta^{m}, m \in \mathbb{N}$, то это предложение допускает сушественное развитие. Пусть $\Omega$ - неограниченная вьпуклая область. Обозначим через $\Theta(G)$ множество направлений звездности области $G$, через $\omega$ - оператор поворота комплексной плоскости на угол $2 \pi / m$. Следующее предложение является аналогом теоремы 4.1 из [19].

ПРЕДЛОЖЕНИЕ 4.7. Пусть для некоторого целого числа $n, 0 \leqslant n<m$, выполняется условие

$$
\bigcap_{k=0}^{n} \Theta\left(\omega^{k} \Omega\right) \neq \varnothing .
$$

Если аннуляторный подмодуль $I \subseteq P(\Omega)$ замкнутого $\pi(D)$-инвариантного подпространства $W \subseteq H(\Omega)$ удовлетворяет условию $\pi-\operatorname{Rank} I \leqslant n+1$, то подпространство $W$ допускает спектральный синтез.

ДокАЗАТЕЛьство. По теореме двойственности достаточно доказать, что аннуляторный подмодуль $I=$ An $W$ является обильным. Обозначим через $J$ замкнутый $\mathbb{C}[l]$-подмодуль в $\mathbf{P}$, порождаемый подмодулем $I$. По следствию $2.3 \pi$-Rank $I=$ $l$-Rank $J \leqslant n+1$.

Значит, по теореме 4.1 из [19] подмодуль $J$ является обильным. По теореме редукции таковым же является подмодуль $I$. Предложение доказано.

Сформулируем основные следствия предложения 4.7.

СЛЕДСТВИЕ 4.3. Пусть $\Omega$ - неограниченная выпуклая область. Тогда всякое замкнутое $\pi(D)$-инвариантное подпространство $W \subseteq H(\Omega)$, аннуляторный подмодуль которого имеет т-ранг 1, допускает спектральный синтез.

СлЕДСТВИЕ 4.4. Пусть $\Omega$ - неограниченная выпуклая область, содержащая угол раствора $2 \pi n / m$. Тогда всякое замкнутое $\pi(D)$-инвариантное подпространство $W \subseteq H(\Omega)$, аннуляторный подмодуль которого имеет т-ранг не больше $n+1$, допускает спектральный синтез. 
СЛЕДСТВИЕ 4.5. Пусть $l(\zeta)=\zeta^{2}, \Omega-$ плоскость, полуплоскость или прямолинейная полоса. Тогда всякое замкнутое $\pi(D)$-инвариантное подпространство $W \subseteq H(\Omega)$ допускает спектральный синтез.

Последнее предложение для случая $\pi(\zeta)=\zeta^{2}$ получено впервые С.Г. Мерзляковым [25].

В заключение исправим досадную ошибку в формулировке леммы 4 из работы [3]. Пусть $\sigma$ - объединение конечного множества $\pi$-слоев.

Лемма. Если $f \in O_{\pi}(\widetilde{\lambda}) \quad \forall \widetilde{\lambda} \subseteq \sigma$, то в некоторой открытой $\pi$-симметричной окрестности $U$ мнохсества $\sigma$ имеет место представление $f=F \circ \pi$, в котором $F$ - функция, голоморфная в некотором открытом полицилиндрическом множестве $V \subseteq \mathbb{C}^{q}$, содержащем $\pi(U)$.

Автор благодарит И. Ф. Красичкова-Терновского за вниманиек работе и обсуждение результатов.

\section{Список литературы}

1. Шишкин А. Б. Спектральный синтез для систем дифференциальных операторов с постоянными коэффициентами // Докл. РАН. 1997. Т. 355. №1. С. 28-30.

2. ШІишкин А. Б. Спектральный синтез для систем диффференциальных операторов с постояннњми коэффициентами. Теорема двойственности // Матем. сб. 1998. Т. 189. №9. C. $143-160$.

3. Красияков-Терновский И. Ф., Шишкин А. Б. Локальное описание замкнутых подмодулей в специальном модуле целых функций экспоненциального типа // Матем. сб. 2001. T. 192. №11. C. 35-54.

4. Красичков-Терновский И. Ф. Инвариантные подпространства аналитических функций. І. Спектральный синтез на выпуклых областях // Матем. сб. 1972. Т. 87. №4. C. $459-489$.

5. Ганнинг P., Росси $X$. Аналитические функции многих комплексных переменных. М.: Мир, 1969.

6. Красичков-Терновский И. Ф. Локальное описание замкнутых идеалов и подмодулей аналитических функций одной переменной. I // Изв. АН СССР. Сер. матем. 1979. Т. 43. №1. C. $44-66$.

7. Чеботарев Н. Г. Теория алгебраических функций. Л.: ОГИЗ, 1948.

8. Красичков-Терновский И. Ф. Спектральньй синтез аналитических функций на системах вьпуклых областей // Матем. сб. 1980. Т. 111(153). № 1. С. 3-41.

9. Красичков-Терновский И. Ф. Локальное описание замкнутых идеалов и подмодулей аналитических функций одной переменной. II // Изв. АН СССР. Сер. матем. 1979. Т. 43. №2. C. 309-341.

10. Красичков-Терновский И. Ф. Спектральный синтез в комплексной области для дифференциального оператора с постоянными коэффициентами. II. Метод модулей // Матем. сб. 1992. Т. 183. №1. С. 3-19.

11. Красичков-Терновский И. Ф. Спектральный синтез в комплексной области для дифференциального оператора с постоянньми коэффициентами. III. Обильнте подмодули // Матем. сб. 1992. Т. 183. №6. С. 55-86.

12. Шишкин А. Б. Локальное описание замкнутых подмодулей в специальном модуле целых функций экспоненциального типа // Матем. заметки. 1989. Т. 46. №6. С. 94-100.

13. ШІишкин А. Б. Спектралњнй синтез для оператора, порождаемого умножением на степень независимой переменной // Матем. сб. 1991. Т. 182. №6. С. 828-848.

14. Красичков-Терновский И. $Ф$. Спектральньй синтез в комплексной области для дифференциального оператора с постоянными коэффициентами. I. Теорема двойственности // Матем. сб. 1991. Т. 182. № 11. С. 1559-1588. 
15. Красичков-Терновский И. Ф. Инвариантные подпространства аналитических функций. II. Спектральный синтез на вьпуклых областях // Матем. сб. 1972. Т. 88. №1. C. $3-30$.

16. Напалков В. В. Уравнения свертки в многомерных пространствах. М.: Наука, 1982.

17. Красичков-Терновский И. Ф. Фундаментальный принцип для инвариантных подпространств аналитических функций. II // Матем. сб. 1997. Т. 188. №6. С. 57-98.

18. Дьедонне Ж., Швари Л. Двойственность в пространствах $(F)$ и $(L F) / /$ Математика. Сб. пер. 1958. Т. 2. № 2. С. 77-107.

19. Красичков-Терновский И. Ф. Спектральньй синтез в комплексной области для дифференциального оператора с постоянньми коэффицциентами. IV. Синтез // Матем. сб. 1992. T. 183. № 8 . C. 23-46.

20. Себаштьян-и-Силва Ж. О некоторых классах локально выпуклых пространств, важных в приложениях // Математика. Сб. пер. 1957. Т. 1. №1. С. 60-77.

21. Эдвардс Р. Функциональный анализ. Теория и приложения. М.: Мир, 1969.

22. Кривошеев A.C., Напалков B. В. Комплексный анализ и операторы свертки // УМН. 1992. T. 47. №6(288). C. 3-58.

23. Schwartz L. Théorie générale des fonctions moyenne-périodiques // Ann. of Math. (2). 1947. V. 48. P. 857-929.

24. Мерзляков С. Г. Инвариантные подпространства оператора кратного диффференцирования // Матем. заметки. 1983. Т. 33. № 5. С. 701-713.

25. Мерзляков С.Г. О подпространствах аналитических функций, инвариантных относительно оператора кратного дифференцирования // Матем. заметки. 1986. Т. 40. №5. C. $635-639$.

Армавирский государственньй педагогический институт

E-mail: Shishkin-home@mail.ru
Поступила в редакцию 26.04 .2002 и 25.06 .2003 